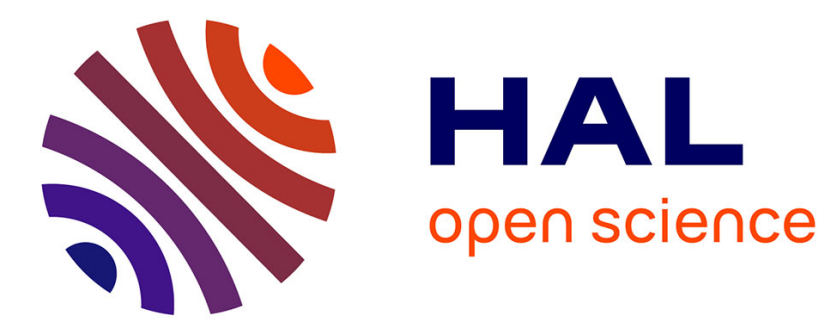

\title{
Private eradication of mobile public bads
}

Christopher Costello, Nicolas Querou, Agnès Tomini

\section{To cite this version:}

Christopher Costello, Nicolas Querou, Agnès Tomini. Private eradication of mobile public bads.

European Economic Review, 2017, 94, pp.23-44. 10.1016/j.euroecorev.2017.02.005 . hal-01594268

\section{HAL Id: hal-01594268 \\ https://hal.science/hal-01594268}

Submitted on 26 Sep 2017

HAL is a multi-disciplinary open access archive for the deposit and dissemination of scientific research documents, whether they are published or not. The documents may come from teaching and research institutions in France or abroad, or from public or private research centers.
L'archive ouverte pluridisciplinaire HAL, est destinée au dépôt et à la diffusion de documents scientifiques de niveau recherche, publiés ou non, émanant des établissements d'enseignement et de recherche français ou étrangers, des laboratoires publics ou privés.

\section{다(1) (2)}

Distributed under a Creative Commons Attribution - ShareAlikel 4.0 International 


\title{
Private eradication of mobile public bads ${ }^{\text {th }}$
}

\author{
Christopher Costello ${ }^{\mathrm{a}}$, Nicolas Quérou ${ }^{\mathrm{b}}$, Agnes Tomini $^{\mathrm{c}}$ \\ ${ }^{a}$ Corresponding Author: Bren School, 4410 Bren Hall, Santa Barbara, CA 93117, and NBER. \\ costello@bren.ucsb.edu.805-893-5802. \\ ${ }^{b}$ CNRS, UMR5474 LAMETA, F-34000 Montpellier, France. querou@supagro.inra.fr \\ ${ }^{c}$ Aix-Marseille University (Aix-Marseille School of Economics): CNRS 83 EHESS, Centre de la \\ Vieille Charité, 2 rue de la charité, F-13002, Marseille, France. E-mail: \\ agnes.tomini@univ-amu.fr
}

\begin{abstract}
We consider analytically the non-cooperative behavior of many private property owners who each controls the stock of a public bad, which can grow and spread across spatial areas. We characterize the conditions under which private property owners will control or eradicate, and determine how this decision depends on property-specific environmental features and on the behavior of other landowners. We show that high mobility or lower control by others result in lower private control. But when the marginal dynamic cost of the bad is sufficiently large, we find that global eradication may be privately optimal - in these cases, eradication arises in the non-cooperative game and is also socially optimal so there is, in effect, no externality.
\end{abstract}

Keywords: Public Bad, Spatial Externality, Invasive Species, Spread, Eradication

JEL classification: H41, Q24, Q57, R12, R14

\section{Introduction}

The management of public bad resources represents a ubiquitous challenge with real-world policy implications. Applications are numerous and include diverse re-

\footnotetext{
in For helpful suggestions we are indebted to Peter Berck, two anonymous referees, Andrew Plantinga, Cathy Kling, Larry Goulder, Esteban Rossi-Hansberg, Klaus Desmet, Daniel Kaffine, Terry Anderson, Leo Simon, Severin Borenstein, Jo Albers, Rebecca Epanchin-Neill, Dan Phaneuf, Corbett Grainger, and seminar participants at UC Berkeley, University of Wisconsin, Colorado State University, Stanford University, Resources for the Future, UC Santa Barbara, PERC, and Paris School of Economics. C. Costello acknowledges the support of the Lone Mountain Fellowship from the Property and Environment Research Center. N. Quérou acknowledges the support from the 'GREEN-Econ' ANR research project (ANR-16-CE03-0005).
} 
sources such as infectious diseases, fire, invasive species, antibacterial resistance, noxious advertising, cyberspace viruses, and aquaculture pathogens, among many others. Controlling these nuisances is complicated by their mobility and renewability since they may grow and spread to surrounding areas in subsequent periods, thus imposing future damages in other locations. While the literature often focuses on socially-optimal management, 1 issues that arise when individual property owners each make decentralized decisions have received less attention for renewable public bads. Indeed, spatial connectivity induced by the mobility of these resource influences private decisions, which collectively can have important consequences for control or eradication across the spatial domain..$^{2}$

Our objective is to provide a general, theoretical treatment of non-cooperation over renewable, mobile public bads. This has important parallels with the canonical transboundary pollution problem where spread of the bad is also a prominent feature. But because our focus is on renewable public bads, we must also account for resource growth. This class of bads, which includes applications such as insect pests on agricultural fields, infectious diseases in countries, and head lice in an elementary school classroom, differs from the transboundary pollution problem in one more fundamental way. For these public bads, eradication is a viable policy option; this type of corner solution has not been the focus of models of cross-jurisdictional emissions of, say carbon or sulfur dioxide, where global (or even local) eradication is not viewed as a policy-relevant option.

With these intended contributions in mind, we note that a growing, though disparate, literature contributes to the issue of public bad management from the spatial perspective. The literature on optimal control of infectious diseases commonly introduces a transmission parameter to capture the rate of spread, but typically does not model the spatial dimension of disease transmission (exceptions are noted in the review by Arino and den Driessche (2006)). An early literature on transboundary pollution, such as acid rain, sheds light on control of pollutants that are not only deposited in the emitting country, but transported by wind, or water, across borders. Calvo and Rubio (2012) provide an informative survey on dynamic models of international environmental agreements when pollution is transboundary, and Jorgensen et al. (2010) focus on dynamic pollution games. However, the transport issue is rarely considered. In van der Ploeg and de Zeeuw (1992) a quadratic welfare function is maximized, considering separate pollution

\footnotetext{
${ }^{1}$ Among others, Lichtenberg and Zilberman (1986) and Archer and Shogren (1996) seek to optimally control a pest population, other biological invasions (Shogren, 2000| Olson and Roy, 2002) or infectious diseases (Wiemer, 1987; Gersovitz and Hammer, 2004). Adda (2015) provides an evaluation of health-related policies relying on cost-benefit analysis.

${ }^{2}$ Brito et al. (1991) and Geoffard and Philipson (1997) focus on the economics of vaccination (but not eradication) and abstract from issues raised by strategic interactions and heterogeneity.
} 
levels in each country, and assuming that a proportion of the emissions spreads to other countries while the rest of the emissions remain at home. They contrast the open-loop Nash equilibrium and the Markov-perfect equilibrium. Mäler and de Zeeuw (1998) explicitly describe a matrix of transport coefficients of emissions and adopt specific functional forms (as do Kaitala and Pohjola (1988) and Escapa and Gutièrrez (1997)), to provide numerical simulations to discuss the gains from cooperation.

A distinction between pollution problems and the resource problems considered here is that the latter class allows for endogenous growth of a renewable public bad. For example, it has become common recently to examine the optimal management of an exotic species that is spatially distributed across the landscape. A sole owner accounts for all spatial connections and optimizes her control efforts across space to derive the optimal design of policies; provided that all externalities are accounted for, such a sole owner achieves the socially-optimal outcome. In this setting some authors focus on the question of prevention vs. control: Leung et al. (2002) find, for zebra mussels, that ex-ante prevention is more efficient than ex-post control, while Burnett et al. (2008) use the case of the Brown Tree snake in Hawaii, after having theoretically characterized the paths of expenditures and damages, to analyze the optimal integrated management of prevention and control. Others contrast long-run solutions from optimal control systems and solutions from a static optimization problem (Finnoff et al. 2010), or uniform vs spatially-optimized policy (Albers et al., 2010). Epanchin-Niell and Wilen (2012) numerically examine optimal policies over a range of spatial and ecological configurations, and stress the influence of these qualitative characteristics on policies. While some purely theoretical works exist (e.g. Blackwood et al. (2010)), most papers conduct numerical simulations either in stylized systems or in systems loosely parameterized by empirical observations because analysis tends to grow in complexity with the spatial domain. These focused numerical applications help establish insights in specific settings, but they also raise more general hypotheses that can be addressed by theory.

A second strand of literature explicitly introduces the non-cooperative nature of private property owners, and emphasizes mechanisms that can be used to induce cooperation. Grimsrud et al. (2008) show that coordination is more likely with low levels of invasion in a two-agent dynamic model. Epanchin-Neill and Wilen (2015) examine how different degrees of cooperation affect invasion and find that the degree of cooperation is related to control costs: less cooperation is required to achieve high control when costs are low relative to damages. Our analysis follows this line of research, but we rather provide a game-theoretical approach with many economic agents instead of conducting numerical analysis of a particular system. Our model also allows for heterogeneous landowners, for example with respect to 
costs, damages, and spread rates. We find that these sources of heterogeneity can significantly alter individual landowner incentives over control or eradication, suggesting that heterogeneity can play an important role in economic outcomes. Our theoretical approach allows us to home-in on the effects of different patterns of spread and infestation on non-cooperative outcomes $3^{3}$ This helps to generalize previous numerical results.

Modeling non-cooperation in a dynamic spatial context is a non-trivial task. As emphasized in the literature on the canonical transboundary pollution model, there are several non-cooperative solution concepts that may be used in dynamic settings. Open-loop Nash equilibrium (OLNE) and Markov-perfect (or feedback) Nash equilibrium (MPNE) are the most commonly used approaches. One critique of the OLNE concept is that it relies on unrealistic information sets and an infinite period of commitment (van der Ploeg and de Zeeuw, 1992). In many settings, it may be more realistic to allow agents to a priori condition the current management decisions on the state variable. Under that assumption, the appropriate noncooperative solution concept is the MPNE, which corresponds to a zero length of commitment (Reinganum and Stokey, 1985) and is subgame-perfect. In our model, we adopt the MPNE solution concept, and thus assume that landowners can condition their current levels of control on the stock of the public bad. Other solution concepts have been used in natural resource management problems, such as the incentive equilibrium or the trigger-strategy equilibrium: the credibility of such strategies often raises important issues (see for instance Breton et al. (2008)); these issues are avoided with the MPNE.

Broadly speaking, the literature on network games provides interesting insights highlighting that players' behaviors are influenced by those around them (Jackson and Zenou, 2014). Indeed, spread rates of a resource, disease transmission parameters, or a network structure represent an adjacency matrix linking agents and serve as a vector to impact their payoffs. This literature helps to characterize how individuals' decisions may depend on interactions across players. For example, biological invasions are often considered a "weakest-link" public good where the level of control is determined by the weakest contributor (Burnett, 2006). Our theoretical framework also has ramifications for "reputation" spillovers, the propagation and transmission of reputation between economic agents. Winfree and McCluskey (2005) analyze reputation as a dynamic common-property resource, and assume that the collective reputation (the common-property good) affects firms' profits. This public good depends on the sum of the level of quality strategically chosen by firms, and the spread of consumer learning on firms' qualities,

\footnotetext{
$\sqrt[3]{\text { Barrett }(2003)}$ develops an insightful model of global disease eradication within a static and homogeneous setting.
} 
following the structure introduced by Shapiro (1982). Consequently the higher the spread level, the more the growth rate of collective reputation increases over time. Following this line, as a negative reputation will injure firms' profits, our study may give theoretical insights on the role of transmission coefficients (spatial parameters) in reputation propagation, and provide a different setting to analyze the emergence of cooperation to control or eradicate bad reputation. Indeed, the cleansing of bad reputation has become big business; large companies (such as "reputationdefender.com") now specialize in eradicating one's negative online reputation. Brandt et al. (2003) also consider a spatial public good to analyze reputation. Much of this literature adopts experimental approaches (e.g. Palfrey and Prisbrey (1996)). Although informative, and suggestive of mechanisms that may be applied more broadly, these papers generally lack theoretical findings so general lessons, and the conditions under which they arise, are difficult to ascertain. This paper contributes by providing a general analysis of decentralized public bad management in a spatial setting.

Our model is a theoretical treatment of the non-cooperative behavior across many heterogeneous landowners controlling a mobile, renewable public bad. One of our focal contributions is to allow for, and specifically analyze the interesting, and policy-relevant corner solution of eradication. We employ the model to derive general results such as the conditions under which control and eradication will emerge, those under which non-cooperation results in a socially optimal pattern of control, and the effects of system characteristics (e.g. the rates of spread) on non-cooperative outcomes. Much of the literature emphasizes the spectrum of policy options to manage public bads, including prevention and cure (Leung et al., 2002) and detection and control (Kaiser and Burnett, 2010), but few authors focus on eradication as a policy option (Olson and Roy, 2002, Burnett et al., 2006). This option is often assumed to be too expensive or too complex (Gersovitz and Hammer, 2005; Regan et al., 2006), yet hundreds of well-known examples of eradication exist, suggesting that real-world circumstances may exist under which it is economically viable. We address this possibility by characterizing the conditions under which eradication is either socially optimal, or is an outcome of non-cooperative behavior across all spatially connected properties.

The class of problems we tackle is most closely aligned with Fenichel et al. (2014), who focus on the decision of a single landowner about the timing of spray intervals to combat a citrus pest; this is framed as an optimal stopping problem. In contrast, our model explicitly treats the dynamic non-cooperative behavior of many landowners who each controls (or possibly eradicates) the public bad on her property. Fenichel et al. (2014) use numerical simulations to shed light on the specific case of a pest infecting a stock of citrus trees. This is helpful for providing numerical results and insights for the problem at hand, but makes it 
difficult to draw general conclusions or to understand the conditions under which those conclusions hold up. In contrast, our model is entirely theoretical, so we are able to provide general results and can prove the conditions under which they will, or will not, hold. This also allows us to examine a range of comparative statics and other results relating to economic and resource heterogeneity. Finally, because we are able to examine if, and under what theoretical conditions, eradication will arise, we address a broader array of possible policy responses than in much of the literature.

Our model contains an arbitrary number of spatially-distinct properties and discrete-time resource dynamics. Here properties could be spatial areas, individual people, countries, or other jurisdictions. Following the tradition to contrast decentralized decisions with optimal management (e.g. Calvo and Rubio (2012), Jorgensen et al. (2010), or Mäler (1989)), we analyze decentralized owners' incentives and the equilibrium behavior across those owners. In our theoretical model we also solve for the socially-optimal control pattern across space and time. While we think this is a contribution in its own right, we regard it primarily as a benchmark case against which to compare decentralized equilibria across non-coordinating property owners.

Aside from developing a tractable analytical framework for addressing mobile public bads, our results make three main analytical contributions. First, we show how the private trade-off between controlling the expansion of a public bad on one's own property and eradicating it depends on the characteristics of its spread. Furthermore, whether global eradication over the entire domain emerges in the decentralized system depends on features such as the degree of patch connectivity. Second, in general we find the intuitive result that non-cooperative property owners will provide too little control of the public bad. This result accords with Fenichel et al. (2014) and is intuitive because private property owners will consider only their local costs and benefits of control, but will disregard the consequences of their actions on adjacent owners. We also show analytically how the extent of this externality is driven by heterogeneity and other features of the problem.

Our third main contribution involves involves inducing cooperation across landowners. Landowner behavior in this model depends both on the current marginal costs of damage and control, and on the discounted future costs; we will refer to the sum of these effects as the marginal dynamic cost; it is what drives marginal control decisions for landowners in our model. If the marginal dynamic cost inflicted by the stock is low, neither the sole owner nor the non-cooperative private property owners will engage in much control, so little is to be gained from cooperation among private owners. In that case, private property delivers a near first-best outcome. But as the marginal dynamic cost increases, private property owners increase their control, but not as much as the sole owner of all properties would have 
liked. Thus, as the marginal dynamic cost grows, so does society's benefit from cooperation among property owners. This intuitive finding suggests that as the size of the externality grows, so does the importance of government intervention (or private ordering) to internalize the externality. But we find that this result holds only for moderate levels of the marginal dynamic cost. If these costs grow enough, then private property owners will eradicate on their own property; for example, you do not tend to observe poison ivy or rattlesnakes in urban backyards, nor do most industrialized countries tolerate flare-ups of infectious diseases such as measles or malaria. These public bads tend to be eradicated even without regard for others' welfare. We show that when eradication arises in the non-cooperative game, then it is also socially efficient. Thus, if the marginal dynamic cost is sufficiently large, the cooperative and non-cooperative solutions converge, and there is no additional value from government intervention or costly cooperative mechanisms across landowners. This finding suggests that government intervention may be justified (to coordinate the actions of private land owners), but only in cases of intermediate marginal dynamic costs. Thus, our third main contribution is to completely characterize the gains from inducing cooperative behavior among the non-cooperative property rights holders. Naturally, to the extent that properties are heterogeneous, the side payments required to achieve cooperation will differ across space. We derive the magnitude of these side payments as a function of damage, cost, spread, and growth.

That eradication may emerge on some, or even all, properties is not just a theoretical curiosity. Numerous examples exist of successful eradications of invasive species on iconic island chains such as the Galapagos or Channel Islands. In New Zealand, Norway rats (which are both ecologically and economically damaging) have been eradicated at relatively small cost (Pimentel, 2011). Among infectious diseases, the most famous global eradication was smallpox in 1980, but country-specific eradications of diseases such as malaria and ebola are numerous. The American West undertook a massive and successful wolf eradication in the early 20th century (which has since been deliberately reversed). And closer to home, most families with school-age children have confronted local eradications of headlice or bedbugs. Some of these eradications required the coordination across individual landowners.4 In other cases, the individual incentives for eradication were sufficient to obviate the need for contracting solutions or government coordination efforts. These casual observations will be formally treated in our analysis.

We organize the paper as follows: The analytical model is introduced in Section 2 and we derive the equilibrium strategies of non-cooperative property owners in Section 3. The sole owner's problem is introduced and solved in Section 4 ,

\footnotetext{
${ }^{4}$ For headlice, a "landowner" could be the individual on whose head the lice have taken hold.
} 
which puts us in a position to compare the decentralized solution with the sole owner's in Section 5. We then calculate the gains from cooperation and discuss the cooperation-inducing side payments in Section 6. We conclude in Section 7. All proofs are found in the Appendix.

\section{A spatially-connected model of a renewable public bad}

The stock of a renewable public bad is spatially distributed. Space is divided into a set $I=\{1, \ldots, N\}$ of mutually exclusive and exhaustive "properties," each of which is assumed to be owned by a single cost minimizing owner. Properties may be heterogenous with respect to biological and economic characteristics, but all economic and ecological characteristics are homogenous within a property. Using a discrete-time model, the stock residing on property $i$ at the beginning of time period $t$ is given by $x_{i t}$ and control efforts undertaken on property $i$ will reduce the stock over the course of that time period. We denote the amount of stock removed (or "controlled") on property $i$ by $h_{i t}$, which leaves a "residual stock" at the end of the period of $e_{i t} \equiv x_{i t}-h_{i t}$. The residual stock grows according to a stock growth function $g\left(e_{i t}\right) !^{5}$ and the resource stock is distributed across the landscape. The fraction of the property $j$ resource stock that moves from property $j$ to property $i$ in a single period is given by $D_{j i}$, where $\sum_{i} D_{j i} \leq 1.6^{6}$ The resulting equation of motion of the resource stock is:

$$
x_{i t+1}=\sum_{j=1}^{N} D_{j i} g\left(e_{j t}\right) ;
$$

Equation (1) is consistent with continuous-time metapopulation models in bioeconomics (e.g. Sanchirico and Wilen (1999)), except that because ours is in discrete time, we must specify the timing of growth and spread. In our model growth occurs prior to spread (for example, a plant might produce seeds before the wind disperses them). Thinking of spread $D$ as an $N \mathrm{x} N$ matrix with typical element $D_{i j}$, the diagonal term $D_{i i}$ is self-retention (the fraction of stock produced in $i$ that remains in $i$ the following period), the other $N-1$ terms in column $i$ (terms

\footnotetext{
${ }^{5}$ Without spread or stock removal, $e_{i t+1}=g\left(e_{i t}\right)$ and we assume the usual conditions: $g(0)=$ $0, g^{\prime}(e)>0, g^{\prime \prime}(e)<0$.

${ }^{6}$ This follows the recent literature from the natural sciences (see, e.g., Nathan et al. (2002), or Siegel et al. (2003)) who model dispersal of passive "Lagrangian particles." An endogenous spread parameter may be a relevant alternative to account for density-dependent process, or situations where agents can affect that process. The latter case introduces an additional control variable (as in Rowthorn et al. (2009)), which has the benefit of introducing the containment policy to keep the bad within some specified area. While this has appeal in some settings, we focus instead on the problem of controlling (or eradicating) the stock of the bad on one's property.
} 
like $D_{3 i}$ or $D_{7 i}$ ) capture immigration from other properties into $i$, and the other $N-1$ terms in row $i$ (terms like $D_{i 1}$ or $D_{i 5}$ ) capture emigration out of $i$ to the other properties. Owner $i$ starts period $t$ by observing the stock $x_{i t}$. The owner can then control the bad on her property, where the cost of control may be property-specific (for example removing invasive mussels may be simpler in shallower water). The marginal cost of control in a property will also depend on the stock size in that property. This captures the so-called stock effect for which the marginal abatement cost is a decreasing function of the stock. When the stock size is $s$, then the marginal control cost is $c_{i}(s)$, where $c_{i}^{\prime}(s)<0$. If the stock at the beginning of the period is $x_{i}$, then the total cost of controlling down to level $e_{i}$ is given by $\int_{e_{i}}^{x_{i}} c_{i}(s) d s$. The residual stock on property $i$ left after control imposes damage on owner $i$, and the damage function may be property-specific (for example, a weed may cause more damage in an agricultural area than in an industrial area). We assume convex damages, so the marginal damage caused by the $s^{\text {th }}$ unit of stock is $k_{i}(s)$, where $k_{i}^{\prime}(s)>0$. Thus, for residual stock $e_{i}$, the total damage that period is $\int_{0}^{e_{i}} k_{i}(s) d s$. Taking all relevant economic variables into account, the period- $t$ cost to owner $i$ of stock, $x_{i t}$, and control, $h_{i t}$ is:

$$
\int_{0}^{x_{i t}-h_{i t}} k_{i}(s) d s+\int_{x_{i t}-h_{i t}}^{x_{i t}} c_{i}(s) d s .
$$

Following the identity $e_{i t} \equiv x_{i t}-h_{i t}$, we can re-write Expression (2) as:

$$
\Phi_{i}\left(x_{i t}, e_{i t}\right)=\int_{0}^{e_{i t}} k_{i}(s) d s+\int_{e_{i t}}^{x_{i t}} c_{i}(s) d s
$$

which is the total period- $t$ cost to property $i$. The first term on the right hand side of Equation 3 is the total damage cost on property $i$ during period $t$ and the second term is the total cost of control during period $t$. Figure 1 illustrates the marginal control cost function $(c(s))$ and marginal damage cost function $(k(s))$ for a particular property in a single time step. If the initial stock in a period is $x$ and control efforts reduce the stock to level $e$, then the total cost of control in that period is given by the light shaded area and the total damage cost in that period is given by the dark shaded area. Management of the public bad will involve choosing the level of control to minimize the sum of control costs and damages, taking all dynamics into account. While the cost to owner $i$ depends only on the stock and control on property $i$, the stock itself will depend on past decisions in all other properties because the resource can move across space (see Equation 1). Thus, all properties are linked together and this is the sense in which we call this resource a public bad. 
Figure 1: Illustration of marginal control cost $(c(s))$ and marginal damage cost $(k(s))$, for a particular property, as a function of the current stock of the public bad, $s$. Shaded regions represent total control cost (light) and total damage cost (dark) of controlling from $x$ down to $e$ over the course of a single period.

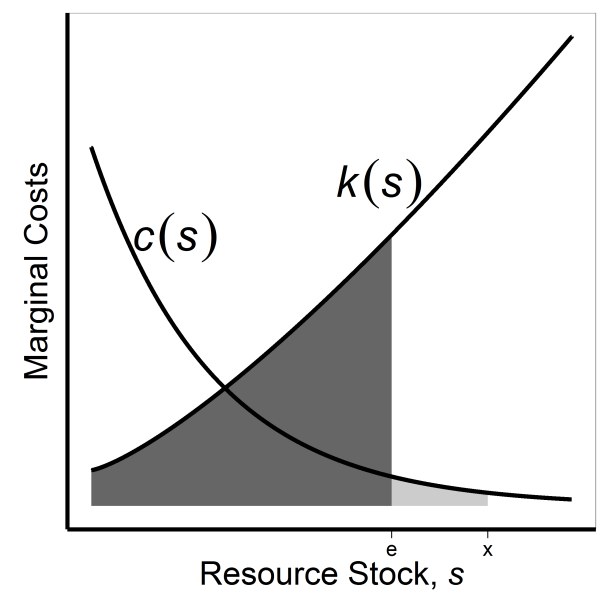

\section{Property owners' strategies}

Here we assume that each of the $N$ property owners makes her own privately optimal decision about how much control to engage in each period. This is a complicated decision for owner $i$ for two reasons. First, owner $i$ 's strategy about how much to control may depend on all current stocks of the public bad and on the strategies applied by all other owners. Second, because the resource grows and moves, owner $i$ 's strategy must account for the fact that less control today implies higher growth and thus higher damage (even on her own property) in future periods. This latter observation allows for a spectrum of management options for owner $i$, from doing nothing to full eradication. In this setting all owners simultaneously choose their level of control, $h_{i t} \geq 0$. Equivalently, owner $i$ can choose $e_{i t} \geq 0$ (since $e_{i t} \equiv x_{i t}-h_{i t}$ and $x_{i t}$ is known at the time of the decision) in order to minimize her present value cost $\hat{V}_{i t}:{ }^{7}$

$$
\hat{V}_{i t}\left(\mathbf{x}_{t}\right)=\min _{e_{i t} \geq 0}\left(\Phi_{i}\left(x_{i t}, e_{i t}\right)+\delta \hat{V}_{i t+1}\left(\mathbf{x}_{t+1}\right)\right)
$$

subject to Equation 1 which defines the state transitions as a function of all owners' controls. We use bold variables to indicate a vector $\left(\right.$ so $\left.\mathbf{x}_{t}=\left(x_{1 t}, \ldots, x_{N t}\right)\right)$ and

\footnotetext{
${ }^{7}$ It turns out to be more mathematically convenient to keep track of the residual stock, $e_{i t}$, rather than the explicit control, $h_{i t}$, so we use $e_{i t}$ as the control variable for property owner $i$. The explicit amount of control, $h_{i t}$, can then simply be backed out.
} 
note that costs are convex in $e_{i t}$. The solution concept in this analysis is Markov Perfect Nash Equilibrium (MPNE). The residual stock decision rule $\left(e_{1 t}, \ldots, e_{N t}\right)=$ $\left(f_{1 t}\left(\mathbf{x}_{t}\right), \ldots, f_{N t}\left(\mathbf{x}_{t}\right)\right)$ is a MPNE if, given the vector of stock levels of the public bad at the beginning of period $t$, at any period $s \geq t\left\{f_{i \tau}\left(\mathbf{x}_{\tau}\right), \tau \geq s\right\}_{i \in I}$ is a solution to the problem 4 above.$^{8}$ As explained in several contributions (see Reinganum and Stokey (1985) among others) there is no commitment issue regarding the owners' decisions, since there is no implicit assumption of agents' binding commitment about actions they will take at future dates when relying on the MPNE concept.

In the following sections, we characterize the owners' optimal management strategies in order to derive the system-wide management outcomes, stock levels, and equilibrium payoffs in the decentralized system. We especially focus on the emergence of control and eradication as equilibrium outcomes. We use the term control to refer to a situation in which a landowner controls some, but not all, of the stock (so $0<e_{i t}<x_{i t}$ ). When discussing eradication, we will distinguish between two important cases: (1) global eradication is a situation in which all properties eradicate the bad, while (2) partial eradication is a situation in which some properties eradicate and others do not.

\subsection{The strategy of control}

We first derive the conditions under which property owners optimally choose to control, but do not eradicate, the public bad. We thus focus on an interior equilibrium $\left(\hat{e}_{i t}>0 \forall i, t\right)$, which is characterized as follows:

Proposition 1. The interior equilibrium of the N-property public bad dynamic game is characterized by residual stock on property $i\left(\hat{e}_{i t}\right)$ given as follows:

$$
k_{i}\left(\hat{e}_{i t}\right)=c_{i}\left(\hat{e}_{i t}\right)-\delta c_{i}\left(\hat{x}_{i t+1}\right) D_{i i} g^{\prime}\left(\hat{e}_{i t}\right)
$$

where $\hat{x}_{i t+1}=\sum_{j=1}^{N} D_{j i} g\left(\hat{e}_{i t}\right)$ denotes the equilibrium stock level at period $t+1$ in property $i$. Moreover, this interior equilibrium is state independent: $\hat{e}_{i t}$ is independent of $\hat{\mathbf{x}}_{t}$ for all $i$.

Here, $\hat{e}_{i t}$ and $\hat{x}_{i t}$ denote the residual stock and resource stock on property $i$, respectively. $\left.\right|^{9}$ The level of control is simply $\hat{h}_{i t}=\hat{x}_{i t}-\hat{e}_{i t}$. Proposition 11 shows that the equilibrium residual stock arises from a trade-off between the current marginal damage (on the LHS) and the marginal dynamic control cost (on the

\footnotetext{
${ }^{8}$ We will use the associated Bellman equations to find such equilibria in several proofs.

${ }^{9}$ The hat indicates the non-cooperative game equilibrium. Sufficient existence conditions are: for any $i \in I k_{i}(0)<\left(1-\delta D_{i i} g^{\prime}(0)\right) c_{i}(0)$ and $k_{i}\left(x_{i 0}\right)-c_{i}\left(x_{i 0}\right)+\delta D_{i i} g^{\prime}\left(x_{i 0}\right) c_{i}\left(D_{i i} g\left(x_{i 0}\right)+\right.$ $\left.\sum_{j \neq i} D_{j i} g\left(x_{j 0}\right)\right) \geq 0$ hold and $\hat{e}_{i t}$ satisfy $g^{\prime}\left(\hat{e}_{i t}\right) \geq \frac{1}{\delta D_{i i}}$. The proof is provided in supplementary material.
} 
RHS). Owner $i$ will control the bad until the current marginal damage is equal to the current marginal cost of removing one additional unit of the stock, mitigated by the discounted future cost implied by an increased stock.

We note also that the strategy of owner $i$ depends on $\hat{x}_{i t+1}$ (because $\hat{x}_{i t+1}$ affects future control costs) ${ }^{10}$ which suggests that owner $i$ 's decision will depend on current decisions of other owners $j$ for whom $D_{j i} \neq 0$ (see Equation 1). If an adjacent owner engages in less control (and so leaves a larger $\hat{e}_{j t}$ ), how will owner $i$ respond? We find a kind of "race to the bottom" emerges, in which less control by owner $j$ implies less control by all connected owners. This consequence of strategic dynamic interactions ${ }^{11}$ among property owners is formalized as follows:

Proposition 2. Provided that all owners control (but do not eradicate) the bad, i.e. $\hat{e}_{i t}>0$, a larger residual stock in one property causes an increase in the optimal residual stock in all connected properties: $\frac{\partial \hat{e}_{i t}}{\partial e_{j t}}>0$, where $D_{j i} \neq 0$.

Proposition 2 show that the incentive to control the public bad increases with the control effort of other owners. The main driver of the result is the stock effect. Owner $i$ must balance current costs of control against current and future benefits of control. The larger is the stock she will inherit from other properties next period, the smaller are her marginal control costs (because $c^{\prime}<0$ ); this effect reduces the incentive to aggressively control today. Thus, as one property owner reduces the stock of public bad on her property, adjacent (or otherwise spatially connected) owners will follow suit. Public bad control is thus a strategic complement, and the strategic reaction to each others' decisions may induce a kind of domino effect. Consequently, this particular game of strategic complements is a spatial analog of the "weaker-link" problem (Cornes, 1993): The level of control in the entire spatial domain is not determined by the lowest individual level effort, but lower control by a single owner will trigger the spread of a spatially mobile public bad. This eventually leads to a loss in welfare across the entire spatial domain. Whether these strategic interactions can lead to eradication remains to be seen. As we will subsequently show, there are cases where full or partial eradication will emerge, even from non-cooperative management.

\footnotetext{
${ }^{10}$ Proposition 1 shows that owner $i$ 's strategy in period $t$ does not depend on $\hat{\mathbf{x}}_{t}$ (intuitively as stock levels at the beginning of the period do not affect the marginal decisions over residual stock), which implies that open loop and feedback rules are identical. The notion of a state independent control strategy is similar to "state separability," a concept used in differential games (Dockner et al. (1985)).

${ }^{11}$ Interactions among properties are strategic because a given owner accounts for other owners' choices when choosing her own privately optimal level of control. The aggregate behavior then impacts the future state of the bad via the equation of motion. In this sense we refer to these as strategic dynamic interactions.
} 


\subsection{The emergence of eradication}

Will private owners ever eradicate the public bad? The majority of the literature focuses on control, and thus neglects whether it is optimal for private agents to eradicate. Indeed, eradication is often considered unrealistic (Simberloff (2009)). For instance, vaccination efforts by some agents may reduce incentives for others to immunize (Anderson and May, 1991), even though it may be socially desirable. Naturally, though, the decision of whether to globally eradicate a public bad will depend on adjacent owners' actions because if they lack control on their property, the likelihood of future infestation may be very high. We thus expect strategic interactions to play an important role in individual eradication decisions.

In order to proceed, we will dissect the optimality condition in Equation (5). That Euler equation defines the first order condition for an interior dynamic optimum. The term $k_{i}\left(\hat{e}_{i t}\right)-c_{i}\left(\hat{e}_{i t}\right)+\delta c_{i}\left(\hat{x}_{i t+1}\right) D_{i i} g^{\prime}\left(\hat{e}_{i t}\right)$ can be thought of as the marginal dynamic cost of the public bad to property owner $i$. Naturally, the property owner would like to set the marginal dynamic cost equal to zero (thus minimizing total cost). But nontrivial corner solution cases exist when it is not possible for that expression to equal zero. We use this fact to analyze the circumstances under which eradication is an outcome of decentralized decisions by property owners. ${ }^{12}$ For example, if even very small stocks impose large damage costs, it turns out that the optimal decision for owner $i$ can be to completely eradicate the stock on her property. If this is the case for all property owners, then global eradication across the entire spatial domain will arise from non-cooperative behavior. These results are summarized as follows:

Proposition 3. Global eradication across the entire spatial domain arises from non-cooperative behavior of property owners if and only if:

$$
\min _{i \in I}\left[k_{i}(0)-\left(1-\delta D_{i i} g^{\prime}(0)\right) c_{i}(0)\right] \geq 0 .
$$

Realistic cases exist in which global eradication in fact does arise, as is suggested by Proposition 3 . Global eradication requires that $\left[k_{i}(0)-\left(1-\delta D_{i i} g^{\prime}(0)\right) c_{i}(0)\right] \geq$ 0 for all properties, $i{ }^{13}$ To gain some intuition, first consider the case in which marginal damage from a small stock $\left(k_{i}(0)\right)$ is large, assuming that $k_{i}^{\prime}(0)-c_{i}^{\prime}(0)$ is positive and sufficiently large so that the second order conditions are satisfied. Provided it is not too costly to eradicate the last units of the stock ( so $_{i}(0)$ is not

\footnotetext{
${ }^{12}$ In the remainder of the analysis, when we mention eradication outcomes, we refer to situations where the public bad is eradicated starting at time $t=0$. We will briefly discuss the implicit role of initial conditions at the end of the analysis.

${ }^{13}$ The proof of Proposition 3 shows that strategies $\left\{f_{i}(.)\right\}_{i \in I}$ defined by $\hat{e}_{i t}=f_{i}\left(\mathbf{x}_{t}\right)=0$ form a MPNE. Since they are time and state independent, there is no difference between open loop and feedback control rules.
} 
too large), eradication will be optimal (this seems to have been the case, e.g., for black and Norway rats in New Zealand). But even if marginal damage from a small stock is small (so the stock must build up before it inflicts any significant damage), it may still be privately optimal to eradicate. To see that, suppose $k_{i}(0)=0$. Then eradication emerges provided that $1<\delta D_{i i} g^{\prime}(0)$, so decentralized eradication is more likely when self-retention $\left(D_{i i}\right)$ is large, intrinsic growth is large $\left(g^{\prime}(0)\right)$, or the discount factor is large $(\delta)$. This interesting result is a consequence of the foresight by the property owner. If she fails to eradicate now, the stock will grow and cause much more damage in subsequent periods; this seems to have been the case, for example, in Australia after some efforts to eradicate mesquite, which has continued to grow and reinvade. 14

Remark 1. Simple conditions ensure that global eradication and control equilibria do not co-exist.15 First, Footnote 9 provides conditions such that the global eradication does not constitute an equilibrium outcome, while the interior equilibrium exists. Second, if for any $j \in I k_{j}(0)>\left(1-\delta D_{j j} g^{\prime}(0)\right) c_{j}(0)$ while there is $i \in I$ such that $c_{i}\left(\sum_{j \neq i} D_{j i} g\left(x_{j 0}\right)\right) \geq \frac{c_{i}(0)-k_{i}(0)}{\delta D_{i i} g^{\prime}(0)}$ an interior equilibrium does not exist, while global eradication is an equilibrium outcome. This requires that the initial stock level in most properties is not too high. We qualify this feature in Section 5 (see Footnote 19).

Cases also exist in which eradication arises on some properties, but not on others; we refer to this as "partial eradication." For example, if property $A$ has high marginal damage from a weed infestation (perhaps it is a native plant nursery) and property $B$ has low marginal damage (perhaps it is rangeland), then this analysis suggests that owner $A$ may find it privately optimal to eradicate the weed on her property while owner $B$ does not. To characterize the conditions under which partial eradication emerges as an equilibrium outcome, we separate the owners into two distinct groups: A group $E$ of $n_{e}>0$ owners who eradicate on their own properties, and a group of remaining owners who optimally choose to only control (but not eradicate) on their properties. As long as some owners fail to eradicate, the public bad will still reside in some of the areas because of growth and spread originating from the control-only properties. The emergence of partial eradication as an equilibrium of non-cooperative behavior is characterized as follows:

Proposition 4. Partial eradication arises from non-cooperative behavior if and

\footnotetext{
${ }^{14}$ For more details, see the Australian government's website: www.environment.gov.au/ biodiversity/invasive/weeds/publications/guidelines/wons/pubs/prosopis.pdf.

${ }^{15}$ The proof of Proposition 3 shows that global eradication Pareto dominates the interior equilibrium. Thus co-existence is not an issue.
} 
only if there exists a group of owners $E \subset I$ such that, in any period $t$ :

$$
\min _{i \in E}\left[k_{i}(0)-c_{i}(0)+\delta D_{i i} g^{\prime}(0) c_{i}\left(\hat{x}_{i t+1}\right)\right] \geq 0
$$

and the remaining property owners control the public bad such that:

$$
k_{j}\left(\hat{e}_{j t}\right)=c_{j}\left(\hat{e}_{j t}\right)-\delta c_{j}\left(\hat{x}_{j t+1}\right) D_{j j} g^{\prime}\left(\hat{e}_{j t}\right) \quad \forall j \notin E .
$$

Proposition 4 can be dissected to extract interesting results on inter-property heterogeneity. For example, properties are more likely to eradicate (not just control) locally if they happen to have higher marginal damage $\left(k_{i}(0)\right)$, lower marginal control cost functions, or higher self-retention rates. Note that for any owner $i \in E$ the corresponding stock level $\hat{x}_{i t+1}=\sum_{j \notin E} D_{j i} g\left(e_{j t}^{e}\right)$ is positive as long as it is connected to another property whose owner only controls the public bad. Intuitively, it is possible that the partial eradication situation described in Proposition 4leaves room for self-consistent transfer payments, where (some) owners from group $E$ are willing to compensate owners from the other group to eradicate the bad. However, this intuition relies on the assumption that global eradication would be socially optimal, which we now analyze.

\section{Socially optimal management of a mobile public bad}

In the spatial dynamic game, property owners consider only the payoffs on their own properties when making privately optimal decisions. By contrast, a sole owner must account for the entire spatial domain when managing the public bad. The sole owner must optimize the spatial and temporal control to minimize the present value of the sum of costs to all properties, subject to the resource dynamics. Written as a dynamic programming equation, the sole owner's problem is to minimize the present value cost $V_{t}$, and the problem is defined as follows:

$$
V_{t}\left(\mathbf{x}_{t}\right)=\min _{\left\{e_{1 t}, e_{2 t}, \ldots, e_{N t}\right\} \geq 0} \sum_{i} \Phi\left(x_{i t}, e_{i t}\right)+\delta V_{t+1}\left(\mathbf{x}_{t+1}\right)
$$

subject to the Equation 1. This appears to be an incredibly complicated problem to solve, particularly as $N$ gets large, because it involves an $N$ dimensional decision where each decision is connected over time via the spread and growth dynamics. But it turns out that this problem can be solved analytically, and that the optimal spatial-temporal control policy can be completely characterized. The convexity of costs in $\mathbf{e}_{t}$ ensure a unique solution.

\subsection{Control as a socially optimal management strategy}

We first consider the case in which control (but not eradication) is optimal on all properties for the sole owner. We denote the vector of socially optimal controls by $\overline{\mathbf{e}}_{t} \equiv\left[\bar{e}_{1 t}, \bar{e}_{2 t}, \ldots, \bar{e}_{N t}\right]$. The socially optimal control policy is given by: 
Proposition 5. The sole owner's optimal control strategy has residual stocks, $\overline{\mathbf{e}}_{t}>0$, characterized as follows

$$
k_{i}\left(\bar{e}_{i t}\right)=c_{i}\left(\bar{e}_{i t}\right)-\delta \sum_{j} c_{j}\left(\bar{x}_{j t+1}\right) D_{i j} g^{\prime}\left(\bar{e}_{i t}\right) .
$$

In a manner similar to the decentralized result (Proposition 1), the sole owner's optimal residual stock results from a trade-off between marginal damage (on the LHS) and the marginal control cost (on the RHS). Again, the marginal control cost is composed of the current marginal control cost and the sum (over properties) of the discounted marginal control cost in the future.

\subsection{Is eradication socially optimal?}

In a manner similar to the decentralized property owners, we can determine the conditions under which global eradication is socially optimal. Intuitively, if damage is very high, or if eradication costs are very low, then it may pay to bear the one-time costs of eradication rather than bear a stream of damages (and costs) in perpetuity. If Condition (10) cannot be met for any $\bar{e}_{i t}>0$, then it is optimal to eradicate the entire resource stock on all properties. There are also conditions under which it is socially optimal to eradicate on some properties but not all. These results are formalized below:

Proposition 6. (a) Global eradication is socially optimal if and only if:

$$
\min _{i \in I}\left[k_{i}(0)-\left(1-\delta D_{i i} g^{\prime}(0)\right) c_{i}(0)+\delta g^{\prime}(0) \sum_{j \neq i} D_{i j} c_{j}(0)\right] \geq 0 .
$$

(b) Eradication across part of the spatial domain is socially optimal if and only if there exists a set of properties $\bar{E} \subset I$ such that, in any period

$$
\min _{i \in \bar{E}}\left[k_{i}(0)-c_{i}(0)+\delta g^{\prime}(0) \sum_{j} D_{i j} c_{j}\left(\bar{x}_{i t+1}\right)\right] \geq 0
$$

and the remaining property owners control the public bad such that, for some $\bar{e}_{j t}>0$

$$
k_{j}\left(\bar{e}_{j t}\right)=c_{j}\left(\bar{e}_{j t}\right)-\delta \sum_{k} c_{k}\left(\bar{x}_{k t+1}\right) D_{j k} g^{\prime}\left(\bar{e}_{j t}\right) \quad \forall j \notin \bar{E} .
$$

The result on global eradication (Proposition 6a) is similar to the case of decentralized management except that here, spatial externalities between all properties are accounted for by the sole owner. Proposition $6 \mathrm{~b}$ characterizes conditions under which it is socially optimal to only partially eradicate. Again, notice that, in any 
property $i \in \bar{E}$, the stock level $\bar{x}_{i t+1}=\sum_{j \notin \bar{E}} D_{j i} g\left(\bar{e}_{j t}\right)$ is positive at the beginning of period $t+1$ provided that it is connected to a property in which it was socially optimal to only control the public bad. While the residual stock level is the same under both management regimes for properties where the public bad is eradicated, it may differ in others.

The results obtained in Sections 3 and 4 imply an interesting dependence of spatial connectivity on the differences between decentralized and socially optimal management; this is further explored in what follows.

\section{Tragedy of the commons, inefficient coordination and spatial connec- tivity}

The literature on decentralized common pool resource management often emphasizes its shortcomings compared to socially optimal management, and these comparisons are often restricted to quantitative comparisons of outcomes. By contrast, we adopt two lines of comparison. First, we analyze the conditions under which the tragedy of the commons emerges (where each owner chooses positive, yet suboptimally low control of the public bad). Second, we characterize cases in which property owners fail to coordinate on the socially-optimal strategies: For example, if they only control, while the sole owner would fully eradicate the mobile public bad. Inspecting Conditions (5) and (10) or Conditions (6) and (7), it is intuitive that spatial connectivity will play an important role in these comparisons.

We first assess how "spread" may exacerbate the tragedy of the commons beyond the deleterious effects arising from strategic interactions, which we have already examined. When no spatial externality exists, $D_{i i}=1$ (so $D_{j i}=0$ for all $j \neq i$ ), and we would expect the decentralized solution, $\hat{e}_{i t}>0$, to equal the socially optimal solution, $\bar{e}_{i t}>0$. If an externality exists, $D_{j i} \neq 0$, and we find that control levels differ under decentralized management, as is summarized below:

Proposition 7. In any period t:

(a) In the absence of spread (so $D_{i j}=0 \forall i \neq j$ ), the decentralized equilibrium is equivalent to the socially optimal policy for all properties, $\hat{e}_{i t}=\bar{e}_{i t}$.

(b) When property $i$ is a pure source, i.e. $D_{i i}=0$, the decentralized level of residuals stock is strictly higher than the sole owner's residual stock for each property, $\hat{e}_{i t}>\bar{e}_{i t}$, when $D_{i j}>0$ for some property $j$.

(c) When $D_{i i} \in(0,1)$, we have, for any property $i, \hat{e}_{i t} \geq \bar{e}_{i t}$.

Proposition 7 confirms that the tragedy of commons emerges under private management, except (intuitively) in the limiting case when there is no spatial connectivity. When a property that harbors the bad is spatially connected to another property, laissez-faire tends to yield a suboptimal level of control; this echoes Fenichel et al. (2014), among others. Moreover, the specifics of spatial 
connectivity do affect owner $i$ 's optimal choice. In the presence of spread, the degree to which owner $i$ 's strategy depends on decisions by owner $j$ will depend both on strategic interactions and on spatial features such as the connectivity. Here we examine the impact of the spread parameters in order to identify how changes in residual stock on property $i$ are driven by changes in the self-retention rates and off-property spread rates. For instance, assume a two-property sinksource system, where property $A$ is the source (so $D_{A A}=0$ and $D_{A B}>0$ ) and property $B$ a sink (so $1 \geq D_{B B}>0$ and $D_{B A}=0$ ). Owner $A$ will thus optimize her control within her property ignoring the mobility of the public bad towards the second property, and thus under control the amount of public bad. On the contrary, owner $B$ will behave like a sole-owner disconnected from her neighbor. By extrapolation to a system with multiple sources and multiple sinks, we may conjecture that the noncooperative outcome should entail larger stock of the public bad than the optimal policy for sources, but that outcomes should be similar in sinks. This special case of unidirectional flow has the advantage that higher control effort levels occur where the spread of contamination is high.

To sharpen intuition, we continue with the two-property case $(N=2)$ and focus on the case where control emerges as the decentralized equilibrium. In that case, there are two self-retention parameters: $D_{A A}$, and $D_{B B}$, and two spread parameters: $D_{A B}$, and $D_{B A}$. The impact of self-retention on property $A, D_{A A}$, and the emigration from property $B$ to $A, D_{B A}$ can both be interpreted as a higher quantity of the public bad so intuition on their effects will be straightforward to garner. A more nuanced question is: How will owner $A$ 's optimal strategy depend on emigration to, and self-retention on, property $B$ (that is, how does $\hat{e}_{A}$ depend on $D_{A B}$ and $\left.D_{B B}\right)$ ? These parameters affect stock on property $B$, and thus due to strategic interactions, will indirectly affect residual stock on property $A$. If Venezuela knows that Colombia is stuck with the Zika virus, how does that influence Venezuela's control efforts? More specifically, if $B$ responds by engaging in less control, then by Proposition 2, owner $A$ may also respond by engaging in less control. All results on the dependence of owner $A$ 's residual stock on the spread of the public bad are summarized as follows:

Proposition 8. Consider the two-property case ( $A$ and $B$ ) and assume an interior solution. There exist $0 \leq \bar{D}_{A}<1$ and $0 \leq \bar{D}_{B}<1$ such that, if either $D_{A A} \geq \bar{D}_{A}$ or $D_{B B} \geq \bar{D}_{B}$, an increase in immigration or emigration (either $D_{B A}$ or $D_{A B}$ ), results in a larger residual stock level on property $A$ :

$$
\frac{\partial \hat{e}_{A t}}{\partial D_{B A}}>0 ; \frac{\partial \hat{e}_{A t}}{\partial D_{A B}}>0
$$

$A$ higher value of self-retention, respectively $D_{A A}$ and $D_{B B}$, results in a lower residual stock level on property $A$ if and only if the respective marginal cost is 
inelastic:

$$
\begin{gathered}
\frac{\partial \hat{e}_{A t}}{\partial D_{A A}}<0 \Leftrightarrow 1>\varepsilon_{1} \text { with } \varepsilon_{1}=-D_{A A} g\left(\hat{e}_{A t}\right) \frac{c_{A}^{\prime}\left(\hat{x}_{A t+1}\right)}{c_{A}\left(\hat{x}_{A t+1}\right)}>0 \\
\frac{\partial \hat{e}_{A t}}{\partial D_{B B}}<0 \Leftrightarrow 1>\varepsilon_{2} \text { with } \varepsilon_{2}=-D_{B B} g\left(\hat{e}_{B t}\right) \frac{c_{B}^{\prime}\left(\hat{x}_{B t+1}\right)}{c_{B}\left(\hat{x}_{B t+1}\right)}>0
\end{gathered}
$$

Proposition 8 derives the equilibrium responses of property owner $A$ to changes in immigration $\left(D_{B A}\right)$, emigration $\left(D_{A B}\right)$, or self-retention to any property $\left(D_{A A}\right.$ or $\left.D_{B B}\right)$. Because we have not required that $D_{A A}+D_{A B}=1$, we can isolate the effects of an increase in an element $D_{A B}$ without requiring a commensurate decrease in $D_{A A}$. Such an increase (say in emigration $D_{A B}$ ) can be thought of as a worsening of the public bad, since it implies that ceteris paribus a larger stock of the bad will arrive on property $B$.

The first part of Proposition 8 shows that residual stock on property $A$ is increasing in both immigration $\left(D_{B A}\right)$ and emigration $\left(D_{A B}\right)$. The intuition is that an increase in $D_{B A}$ is as if owner $B$ now engages in less control, since more resource moves toward property $A$. This consequently entices owner $A$ to raise the stock on her own property. The effect of higher $D_{A B}$ on optimal stock in $A$ is driven by the strategic interactions between owners, following Proposition 2, so an increase in $D_{A B}$ causes owner $A$ to raise the stock on her own property.

Behavior consistent with these results was observed during the Ebola outbreak in 2014. The US Centers for Disease Control issued travel warnings to and from West Africa in July, 2014, which effectively decreased both immigration and emigration of the public bad. This partly motivated aggressive control of the outbreak. By July 2015, new cases of Ebola had been reduced to less than 10\% of the earlier rate 16

Analyzing the effects of self-retention $\left(D_{A A}\right.$ and $\left.D_{B B}\right)$ also yields insights, though this becomes more complicated. Here, whether $\hat{e}_{A t}$ will increase or decrease in response to a rise in $D_{A A}$ (or $D_{B B}$ ) will depend on the elasticity of control costs. The net change is the sum of a direct (positive) effect, due to a marginal increase $c_{A}\left(\hat{x}_{A t+1}\right)$, and an indirect (negative) effect on next period stock, $D_{A A} c_{A}^{\prime}\left(\hat{x}_{A t+1}\right) g\left(\hat{e}_{A t}\right)$. If the marginal cost of control is relatively flat (so $c^{\prime}(.) \approx 0$ ), then owner $A$ will engage in more control if $D_{A A}$ is larger. This makes intuitive sense: If a pest population is more likely to persist on one's property, then it seems intuitive that the owner would engage in more control compared to a case in which it is likely to quickly move off of one's property. As a consequence of strategic dynamic interactions, a similar result emerges regarding owner $A$ 's

\footnotetext{
${ }^{16}$ See the Center for Disease Control Ebola control timeline: http://www.cdc.gov/about/ ebola/timeline.html.
} 
response to an increase in $D_{B B}$. But these results can be flipped if marginal cost is sufficiently steep; the proof of Proposition 8 (in the Appendix) spells this out in detail. When spread is such that $\sum_{j} D_{i j}=1$ results remain largely consistent: they may differ only for large values of marginal cost elasticities 17 A final remark is that spatial parameters and growth affect control strategies in different ways. For example, it can be shown that a faster resource growth rate on property $A$ gives rise to a lower non-cooperative residual stock on both $A$ and $B{ }^{18}$

If it is socially optimal to globally eradicate and global eradication emerges under laissez-faire, then there is no tragedy of the commons. However, whether another form of inefficiency may exist, where private owners under laissez-faire coordinate on the "wrong" strategy (the suboptimal one), is an open question. We now turn to the issue of comparing the type of optimal strategies induced by decentralized and socially optimal management. We investigate the conditions under which global eradication can emerge in both the decentralized and socially optimal settings, and how these conditions depend on spatial connectivity. A useful first result is summarized below:

Lemma 1. If global eradication emerges as a decentralized solution, then global eradication is socially optimal.

Lemma 1 shows that it is possible that there is consistency between the control by decentralized private owners and the optimal control by a sole owner. If decentralized property owners all find it privately optimal to locally eradicate the public bad (e.g. because the damages they faced were sufficiently large to justify the cost of eradication), then global eradication is also socially optimal. It turns out that this will always be the case for sufficiently large values of $D_{i i} g^{\prime}(0)$ (when $\left.1<\delta D_{i i} g^{\prime}(0)\right)$, summarized by the following corollary:

Corollary 1. If self retention is sufficiently high on all properties, that is:

$$
\min _{i \in I} D_{i i} \geq \frac{1}{\delta g^{\prime}(0)}
$$

global eradication is socially optimal and emerges from non-cooperative behavior.

\footnotetext{
${ }^{17} \mathrm{~A}$ section is provided in the Appendix that spells out how Proposition 8 changes when we restrict spread so $\sum_{j} D_{i j}=1$.

${ }^{18}$ To obtain this result, the equation of motion (1) can be amended as $x_{i t+1}=$ $\sum_{j=1}^{N} D_{j i} g\left(e_{j t}, \alpha_{j}\right)$ with $\alpha_{j}$ patch-specific parameters reflecting resource growth (e.g. intrinsic rate of growth or carrying capacity). Assuming that $\frac{\partial g(x, \alpha)}{\partial \alpha}>0$ and $\frac{\partial^{2} g(x, \alpha)}{\partial x \partial \alpha}>0$ hold, the reasoning in Proposition 8 yields $\frac{\partial \hat{e}_{A t}}{\partial \alpha_{A}}<0$ and $\frac{\partial \hat{e}_{A t}}{\partial \alpha_{B}}<0$, which both differ from the effects of immigration or emigration and do not depend on the elasticity of marginal costs.
} 
Corollary 1 implies that if all self-retention parameters are sufficiently large, then there will be no tension between socially optimal and private incentives. 19 Conversely, it may often be the case that global eradication is socially optimal, but does not arise from decentralized owners' decisions. This could be the case either if all owners privately choose control, or if eradication arises on some properties, but not on others. For example, if property $A$ has high marginal damage from an invasive weed and property $B$ has low marginal damage, then this analysis suggests that owner $A$ may find it privately optimal to exterminate the weed on her property while owner $B$ does not. But even in that case (when one decentralized owner eradicates and another does not), it may be socially optimal to fully eradicate on all properties (for example if $A$ is downwind of $B$ ). This result could arise because failing to eradicate on property $B$ eventually causes damage on property $A$, which diminishes social welfare. These results are summarized as follows:

Corollary 2. Assume that global eradication is socially optimal (Condition 11 is satisfied), then it will never arise from decentralized management if:

$$
\max _{i \in I}\left[k_{i}(0)-c_{i}(0)\left(1-\delta D_{i i} g^{\prime}(0)\right)\right]<0 .
$$

Corollary 2 characterizes situations under which a tension arises between socially optimal management and private incentives: while it is socially optimal to eradicate in all regions, private owners may choose a different policy. This result will enable us to delve into the cases for which eradication is biologically and/or economically feasible; we focus on the role of spatial parameters:

Proposition 9. Suppose global eradication is socially optimal, and denote by $i$ a property with sufficiently low self-retention $\left(D_{i i}<\frac{1}{\delta g^{\prime}(0)}\right)$. Then the effect of spatial parameters on the emergence of eradication is summarized as follows:

(a) An increase in spread $D_{i j}$ (where $i \neq j$ ) makes the emergence of global eradication less likely under decentralized management.

(b) Provided that self retention remains lower than $\frac{1}{\delta g^{\prime}(0)}$, an increase in self retention makes the emergence of global eradication less likely under decentralized management.

The effect of emigration is straightforward. Using (15), global eradication is not a decentralized outcome when for instance $c_{i}(0)>\frac{k_{i}(0)}{1-\delta D_{i i} g^{\prime}(0)}$ holds for property owner $i$. An increase in emigration does not alter this condition, which means

\footnotetext{
${ }^{19}$ Corollary 1 qualifies the conditions of Remark 1 as follows. If there is a property $i \in I$ such that either $c_{i}(0) \leq k_{i}(0)$ or $\sum_{j \neq i} D_{j i} g\left(x_{j 0}\right) \leq c_{i}^{-1}\left(c_{i}(0)-k_{i}(0)\right)$ is satisfied, then an interior equilibrium does not exist. This is so if there is a property $i$ such that $x_{k 0} \leq$ $g^{-1}\left(\frac{1}{\sum_{j \neq i} D_{j i}}\left(c_{i}\right)^{-1}\left(c_{i}(0)-k_{i}(0)\right)\right)$ holds with $x_{k 0}=\max _{j \neq i} x_{j 0}$.
} 
that the incentives of decentralized property owners do not change. However, such an increase in emigration increases the incentive for the sole owner to eradicate. Thus, this enlarges the set of cases where a tension arises between the two types of management. But the effect of self retention is harder to intuit. Corollaries 1 and 2 allow to deduce that when all properties except one (say $i$ ) are characterized by sufficiently high values of self retention, then an increase in the value of self retention on property $i$ may have two opposing effects. If the increase is such that self retention is now higher than the threshold value, then Corollary 1 implies that it has a positive effect as it removes the potential tension between socially optimal and private incentives. However, if the initial value is so low that the increase is not sufficient to move it over the threshold, then the effect is negative as it enlarges the set of cases where global eradication fails to emerge under decentralized management. This can be deduced from Condition 15 in Corollary 2, These results can be used to assess whether we might expect strong consistency between socially and decentralized management or tensions arising due to strategic behavior.

\section{Cooperation with side-payments}

So far, we have analyzed the decentralized decisions of property owners who are harmed by a public bad that moves across space, and we have contrasted that case with the optimal solution of a sole owner who can perfectly anticipate the spatial migration of the public bad, and who can perfectly target control efforts across space. An obvious result is that there are cases in which a tension arises between these two types of management. We first provide general insights on the potential gains from cooperation:

Proposition 10. (a) If for all $i \in I k_{i}(0) \geq c_{i}(0)\left[1-\delta D_{i i} g^{\prime}(0)\right]$ (large marginal dynamic costs) the gains from cooperation are equal to zero;

(b) If for all $i \in I$ we have $-\delta g^{\prime}(0) \sum_{j \neq i} D_{i j} c_{j}(0)<k_{i}(0)-c_{i}(0)+\delta D_{i i} g^{\prime}(0) c_{i}(0)<$ 0 (moderate marginal dynamic costs), the gains from cooperation are greater than

$$
\frac{1}{1-\delta}\left[\sum_{i=1}^{N} \hat{e}_{i}\left\{k_{i}(0)-c_{i}(0)+\delta g^{\prime}(0)\left[\sum_{l=1}^{N} D_{j l} c_{l}(0)\right]\right\}\right]
$$

(c) If for all $i \in I k_{i}(0)-c_{i}(0)+\delta D_{i i} g^{\prime}(0) c_{i}(0)<-\delta g^{\prime}(0) \sum_{j \neq i} D_{i j} c_{j}(0)$ (low marginal dynamic costs), the gains from cooperation are smaller than

$$
\frac{1}{1-\delta} \sum_{i=1}^{N}\left(\hat{e}_{i}-\bar{e}_{i}\right)\left[c_{i}(0)-k_{i}(0)\right],
$$

Where $\hat{e}_{i}\left(\bar{e}_{i}\right)$ denote the interior equilibrium strategies (socially optimal controls) corresponding to the different cases. 
Proposition 10 highlights an interesting link between the marginal dynamic cost and the potential gains from cooperation. When the marginal dynamic cost is large (case $(a)$ ), there are no gains from cooperation because there is no difference between the "best" equilibrium outcome and the social optimum. In cases $(b)$ and (c) these gains correspond to the saving in present value costs between interior equilibrium outcomes and the social optimum. For given values of $\hat{e}_{i}$ and $\bar{e}_{i}$ the (lower) bound in (16) would be larger than the (upper) bound in (17): gains are relatively larger in the second case than in the third one. They get small (in absolute value) in the third case if either $c_{i}(0)$ and $k_{i}(0)$ or $\hat{e}_{i}$ and $\bar{e}_{i}$ get close for any $i \in I$. Gains get larger (in absolute value) in the second case as $k_{i}(0)-c_{i}(0)+\delta g^{\prime}(0) \sum_{j} D_{i j} c_{j}(0)$ gets larger. This suggests that the gains from cooperation might be larger for moderate values of the marginal dynamic costs.

Looking at the gains from cooperation naturally raises the question of what institutions can help transition from the decentralized solution to the socially optimal solution 20 The case where partial eradication arises from decentralized behavior, but where global eradication is socially optimal, provides a convenient case to assess the potential of monetary transfers to achieve the socially-optimal outcome. Indeed, in that case, we need only provide incentives to a restricted set of owners. The focus of this section is to assess the impact of spatial characteristics on the size of the benefit from cooperation.

To sharpen the findings, we return to the case of two properties $(A$ and $B)$. We ask when a monetary transfer from owner $A$ to owner $B$ is Pareto-Improving, that is, it makes both owners better off compared to the (no-transfer) decentralized management outcome. While we have not explicitly modeled transaction costs, it seems reasonable to assume that the larger is the potential benefit from cooperation, the more likely it is that transaction costs can be overcome (Demsetz, 1967). Thus, we would like to explore the conditions under which we might expect a large, or small, benefit from global cooperation over the control of this spatially-mobile public bad.

We first re-write Conditions (7) and (8) for partial eradication to occur in the case of two regions. The stock will be eradicated only on property $A$ if and only if:

$$
k_{A}(0)-c_{A}(0)+\delta g^{\prime}(0) D_{A A} c_{A}\left(D_{B A} g\left(\hat{e}_{B t}\right)\right) \geq 0
$$

where $\hat{e}_{B t}>0$ is characterized implicitly by the following equation:

$$
k_{B}\left(\hat{e}_{B t}\right)-c_{B}\left(\hat{e}_{B t}\right)+\delta D_{B B} g^{\prime}\left(\hat{e}_{B t}\right) c_{B}\left(D_{B B} g\left(\hat{e}_{B t}\right)\right)=0 .
$$

Condition (19) means that owner $B$ chooses to control, leaving a residual stock

\footnotetext{
${ }^{20}$ One mechanism that holds promise for public goods (but to our knowledge has never been analyzed for public bads) is "unitization" (Kaffine and Costello, 2011).
} 
$\hat{e}_{B t}>0$. Condition 18 means that owner $A$ eradicates the public bad each time period. ${ }^{21}$ Since $c_{A}^{\prime}(\cdot)<0$ and $D_{B A}>0$, the incentives of owner $A$ are weaker than under eradication defined by Condition (6).

If Equations (18)-19 hold, owner $A$ eradicates every time period, while owner $B$ only controls the public bad on her property. Since this holds every time period, the corresponding infinite horizon payoffs $\hat{\Pi}_{A}$ and $\hat{\Pi}_{B}$ for owners $A$ and $B$ are defined as follows:

$$
\begin{aligned}
& \hat{\Pi}_{A}=-\left[\int_{0}^{0} k_{A}(s) d s+\int_{0}^{x_{A 0}} c_{A}(s) d s+\frac{\delta}{1-\delta}\left(\int_{0}^{0} k_{A}(s) d s+\int_{0}^{D_{B A} g\left(\hat{e}_{B}\right)} c_{A}(s) d s\right)\right](20) \\
& \hat{\Pi}_{B}=-\left[\int_{0}^{\hat{e}_{B}} k_{B}(s) d s+\int_{\hat{e}_{B}}^{x_{B 0}} c_{B}(s) d s+\frac{\delta}{1-\delta}\left(\int_{0}^{\hat{e}_{B}} k_{B}(s) d s+\int_{\hat{e}_{B}}^{D_{B B} g\left(\hat{e}_{B}\right)} c_{B}(s) d s\right)\right]
\end{aligned}
$$

Conversely, as the socially optimal outcome is characterized by global eradication, the corresponding payoffs of each owner are defined as follows:

$$
\begin{aligned}
& \bar{\Pi}_{A}=-\int_{0}^{x_{A 0}} c_{A}(s) d s \\
& \bar{\Pi}_{B}=-\int_{0}^{x_{B 0}} c_{B}(s) d s
\end{aligned}
$$

Now, consider the possibility of owner $A$ making a payment to owner $B$ to reduce the residual stock in her property. The transfer will be feasible if there exist positive gains from cooperation, that is, if the sum of payoffs (22) and (23) resulting from cooperation exceeds the sum of payoffs (20) and (21) resulting from decentralized management. This is indeed the case, as highlighted by:

Proposition 11. Consider the two-property case (A and B). Assume that global eradication is socially optimal (that is, the cooperative outcome) and that Conditions 18 and 19 are satisfied. Then, there are positive gains from cooperation:

$$
S=\bar{\Pi}_{A}+\bar{\Pi}_{B}-\hat{\Pi}_{A}-\hat{\Pi}_{B}>0,
$$

where $S$ is the overall surplus or gain from cooperation. Proposition 11 shows that, under certain conditions with decentralized management, the overall surplus is positive, but only property owner $A$ gains from cooperation, while property owner $B$ loses from it. The owner of property $A$ has incentives to compensate the owner of property $B$, considered as the weakest-link, to reduce the residual

\footnotetext{
${ }^{21}$ The re-writing follows from Proposition 4 Due to the expressions $\sqrt{18}$ and $(19)$ and the fact that economic costs, growth and spread are time independent, the optimal choice of owner $B$ is time and state independent (see Proposition 1 . In other words $\hat{e}_{B t}$ can simply be written $\hat{e}_{B}$.
} 
stock on her property. Thus, an appropriate transfer payment might be used to induce owner $B$ to engage in additional control, thus lowering her residual stock level, because it would benefit the adjacent owners. Here a straightforward sidepayment can implement the socially optimal outcome, as the following Proposition shows.

Proposition 12. Consider the two-property case ( $A$ and $B)$. Assume that global eradication is socially optimal (that is, the cooperative outcome) and that Conditions 18 and 19 are satisfied. Consider the following side-payment scheme: at period $t=0$, owner $A$ makes a (one-shot) fixed payment $\gamma$ to owner $B$, and in all periods $t \geq 0$ there is an additional variable transfer (from $A$ to $B$ ) $\tau\left(e_{A t}, e_{B t}\right)$ defined by

$$
\tau\left(e_{A t}, e_{B t}\right)=\left[k_{A}(0)-c_{A}(0)+\delta D_{A A} g^{\prime}(0) c_{A}(0)\right] e_{A t}+\left(k_{B}(0)-c_{B}(0)\right) e_{B t}
$$

Then there exist values of $\gamma$ such that this side-payment scheme induces global eradication.

As Proposition 12 highlights, the form of appropriate side-payment schemes is quite simple. The proof of this Proposition shows several features of the scheme. First, there is a one-shot payment from owner $A$ to owner $B$ in the initial period. Second, the variable side-payment does play a role by ensuring that the owners have appropriate incentives to adhere to strategies that yield the global eradication outcome. Finally, there is an interval of feasible values of the fixed payment. For example, within that range, one that would satisfy fairness properties would result in equal sharing of the surplus:

$$
\gamma=\frac{\left(\bar{\Pi}_{A}-\hat{\Pi}_{A}\right)-\left(\bar{\Pi}_{B}-\hat{\Pi}_{B}\right)}{2}
$$

Other values of the fixed payment are feasible. For example, $\gamma=\hat{\Pi}_{B}-\bar{\Pi}_{B}$ is the smallest payment that would satisfy the owners' participation constraints. The actual amount to be transferred depends on the spatial characteristics. The next proposition examines how the surplus from cooperation and the fixed payment depend on these features.

Proposition 13. Consider the two-property case (A and B). Assume that global eradication is socially optimal (that is, the cooperative outcome) and that Conditions (18) and (19) are satisfied. Then, the following conclusions hold:

(a) The gains from cooperation are increasing in emigration from property $B$ to property $A\left(D_{B A}\right)$.

(b) The gains from cooperation are increasing in self-retention $\left(D_{B B}\right)$ provided marginal cost is elastic. 
(c) The fixed payment, $\gamma$ characterized by (26), is increasing in emigration from property $B$ to property $A\left(D_{B A}\right)$.

(d) The fixed payment, $\gamma$ characterized by (26), is decreasing in self-retention $\left(D_{B B}\right)$ provided marginal cost is in elastic, otherwise the effect is ambiguous.

Proposition 13 shows that the gains from cooperation and the fixed payment increase in spread $\left(D_{B A}\right)$. An increase in $D_{B A}$ affects property $A$, as a larger invasion arises on this property (which results in a higher stock in the long run), while the situation in property $B$ remains unchanged. Non-cooperative control costs thus marginally increase in the long run in property $A$, which increases the difference between cooperation and non-cooperation on that property. This effect increases the gains from cooperation overall, and the incentives to make a transfer to achieve global eradication. Second, an increase in self-retention $\left(D_{B B}\right)$ has a slightly more complicated effect, as it impacts control costs in both properties. There is a direct effect on property $B$, because a higher fraction of the public bad remains on that property, while the same fraction emigrates out of it. This results in higher non cooperative control costs. Property $A$ is also indirectly affected due to the spread from $B$. Indeed, higher self-retention $D_{B B}$ results in a higher residual stock in property $B$ when the marginal cost is elastic (see Proposition 8), and a fraction of this larger residual stock is then transported to property $A$. This increases marginal control costs in property $A$ in the long run. Consequently, the difference between cooperative gains and non-cooperative gains is higher for both properties and overall. Regarding the fixed payment, when the marginal cost is inelastic (see Proposition 8), the impact of a higher self-retention $D_{B B}$ is negative, reducing the impact in area $A$, which reduces owner $A$ 's incentives to compensate owner $B$. Under a quite elastic marginal cost, the effect becomes ambiguous, depending on the balance between the impact on the difference between cooperation and noncooperation on property $A$ versus the impact on this same difference on property $B$. When the effect on property $A$ is the largest, an increase in self-retention $\left(D_{B B}\right)$ intuitively increases the fixed payment, $\gamma$.

Even in the absence of side-payments, there are likely other mechanisms that, under the right circumstances, could lead to cross-property cooperation. Barrett (1994) provides the seminal analysis on international environmental agreements (IEAs) and finds that unless the welfare wedge between full cooperation and noncooperation is small, IEAs are unlikely to be self-enforcing for more than a handful of countries. Kaffine and Costello (2011) define a unitization mechanism where property owners who extract a mobile renewable resource can share profits such that even an arbitrarily large number of owners may voluntarily adhere to the cooperative agreement, though they rely heavily on harsh punishment strategies for defectors. One of the key findings of this paper is that even in the absence of any coordination scheme, owners may find it privately optimal to undertake a 
socially optimal eradication of the public bad.

\section{Conclusion}

We have developed and analyzed a model of a renewable public bad resource, such as an invasive species or infectious disease that can move across space. Decentralized property owners undertake costly control to reduce damage on their own properties, and because the resource is mobile, this control has consequences for all other property owners. The resulting externality induces a spatial-temporal game between the property owners who will each act strategically given the behavior of other owners. Our first contribution is to completely characterize the equilibrium strategy of each owner and the resulting effects on stock and control of the public bad across space. We also solve for the socially optimal level of control across space and show that it always (weakly) exceeds the level of control undertaken by decentralized owners.

A key focus of our analysis is on the conditions under which eradication is undertaken by decentralized owners and/or is desired by the sole owner. We find that there is often consistency between these - realistic cases exist in which all decentralized owners will eradicate the stock on their properties; in these cases the sole owner would also choose to eradicate, so no policy intervention is warranted. But cases also exist in which one or more decentralized owner fails to locally eradicate (even though it is socially desirable). In such cases, side-payments can induce appropriate control, and we characterize the features of the problem that lead to large or small potential gains from this kind of side payment.

Our results also imply an interesting role of initial conditions on decentralized and optimal control of a spatially-connected public bad. If the initial invasion is sufficiently large on all properties, then all property owners will control to their optimal levels immediately and the resulting level of residual stock will be independent of the initial invasion size. But if the initial invasion is large on some properties but small on others, we can obtain a striking result. Consider the twoproperty case and assume the initial invasion occurs only on property $A$ (not on property $B$ ). In that case, it will be efficient for $A$ to control more than she would have had the invasion also extended to property $B .{ }^{22}$ Thus, reasonable conditions exist under which a sole owner would find it optimal to aggressively control (or even eradicate) a "small" initial invasion, even though it would be optimal to only weakly control a more spatially-extensive invasion. Similar dynamics play out under laissez-faire.

\footnotetext{
${ }^{22}$ To see this, inspect Equation $\sqrt{10}$ for property $A$. Since there is no initial invasion on property $B$, the next period's stock $x_{A t+1}$ is small. Because $c_{A}^{\prime}(s)<0$, this drives down the optimal residual stock in $A$. A formal statement is provided in the Appendix.
} 
To obtain sharp analytical results of this spatial-temporal game has required making many simplifying assumptions. We modeled marginal damage on property $i$ as a function of resource stock on property $i$, which depends on the previous period's stock in all properties and the spread from those properties to property $i$. A more complicated version of damage would allow for damage in period $t$ to depend on how much damage had been caused in previous periods, as it is often modeled in the acid rain literature (see the survey of Calvo and Rubio (2012)). We modeled the marginal control cost as a decreasing function of the stock. This mirrors the approach taken in bioeconomics but differs from most contributions in transboundary pollution management where control costs depend on emissions flows. Here the higher is the local stock, the smaller is the marginal cost of abatement. While our approach seems to fit most applications, an extension could allow for marginal cost to also depend explicitly (not just implicitly) on the quantity removed. Regarding the spread, we have assumed that the fraction of the stock that spreads from property $i$ to property $j$ is constant. An extension could allow for the spread to depend on the density of the stock in both areas. While these changes would complicate the solution to our model, we think they are unlikely to overturn the main findings of this paper. But these are fertile opportunities for applications of this work.

Our approach fundamentally assumes that the resource is a public bad for all property owners. An interesting extension would allow the resource to be a public good for a subset of owners. For example, wolves may be a "bad" for ranchers and a "good" for conservationists. This type of public good has been analyzed by Weitzman (2015). This would enable us to consider conflicts of interest between those who want to conserve the resource, and those who will impede the provision of the public good. Moreover, at a first glance, we expect that the structure of strategic interaction of the group benefiting from the public good might be different, such that the control strategy (Proposition 2) might become a strategic substitute, thus inducing interesting, and yet unexplored, dynamics. While we have not explicitly analyzed public goods here, the structure of our existing model should suffice in that case, where $k_{i}(s)<0$ for a property owner who places positive value on the resource stock. The main derivations still carry through, where properties with positive value simply do not ever remove any stock.

Overall, our results suggest an interesting general result about the gain from coordination among decentralized property owners. If the marginal dynamic cost of the public bad is small, then decentralized owners choose a level of control that is lower than, but approximately equal to, the control that would be chosen by a sole owner. In those cases, the gain from coordination of decentralized owners is likely to be small. If the marginal dynamic cost is moderate, an interior solution is likely to obtain under which some control will be undertaken by the 
decentralized property owners, but that this control will fall well short of what would be chosen by the sole owner. In these cases, the gains from coordination are large. But when marginal damage is large, decentralized owners will choose to eradicate on their own property and in those cases, global eradication is also socially optimal. In such cases, there is no gain from coordination, though this hinges on eradication being a viable policy option. Taken together, these results suggest that the gain from coordination among decentralized owners is largest for an intermediate level of public bads, which may run counter to intuition and may be suggestive of cases when government intervention or coordination schemes are most economically relevant.

\section{References}

Adda, J. (2015). Economic activity and the spread of viral diseases: Evidence from high frequency data. Quarterly Journal of Economics forthcoming.

Albers, H. J., C. Fischer, and J. N. Sanchirico (2010). Invasive species management in a spatially heterogeneous world: Effects of uniform policies. Resource and Energy Economics 32, 483-499.

Anderson, R. M. and R. M. May (1991). Infectious diseases of humans: dynamics and control. Oxford University Press.

Archer, D. and J. Shogren (1996). Endogenous risk in weed control management. Agricultural Economics 14, 103-122.

Arino, J. and P. V. den Driessche (2006). Disease spread in metapopulations. Nonlinear dynamics and evolution equations 48, 1-13.

Barrett, S. (1994). Self-enforcing international environmental agreements. Oxford Economic Papers 46, 878-894.

Barrett, S. (2003). Global disease eradication. Journal of the European Economic Association 1(2-3), 591-600.

Blackwood, J., A. Hastings, and C. Costello (2010). Cost-effective management of invasive species using linear-quadratic control. Ecological Economics 69(3), $519-527$.

Brandt, H., C. Hauert, and K. Sigmund (2003). Punishment and reputation in spatial public goods games. Proceedings of the Royal Society of London B: Biological Sciences 270 (1519), 1099-1104. 
Breton, M., A. Sokri, and G. Zaccour (2008). Incentive equilibrium in an overlapping-generations environmental game. European Journal of Operational Research 185, 687-699.

Brito, D. L., E. Sheshinski, and M. Intriligator (1991). Externalities and compulsory vaccinations. Journal of Public Economics 45(1), 69-90.

Burnett, K. (2006). Introductions of invasive species: Failure of the weaker link. Agricultural and Resource Economics Review 35, 21-28.

Burnett, K., S. D'Evely, B. Kaiser, P. Nantamanasikarn, and J. Roumasset (2008). Beyond the lamppost: optimal prevention and control of the brown tree snake in hawaii. Ecological Economics 67(1), 66-74.

Burnett, K., B. Kaiser, B. Pitafi, and J. Roumasset (2006). Prevention, eradication, and containment of invasive species: Illustration from hawaii. Agricultural and Resource Economics Review 35(1), 63-77.

Calvo, E. and S. Rubio (2012). Dynamic models of international environmental agreements: A differential game approach. International Review of Environmental and Resource Economics 6(4), 289-339.

Cornes, R. (1993). Dyke maintenance and other stories: Some neglected types of public goods. Quarterly Journal of Economics 108(1), 259-271.

Demsetz, H. (1967). Toward a theory of property rights. The American economic review, 347-359.

Dockner, E., G. Feischtinger, and S. Jorgensen (1985). Tractable classes of nonzerosum open loop nash differential games: theory and examples. Journal of Optimization Theory and Application 45(2), 179-187.

Epanchin-Neill, B. and J. Wilen (2015). Individual and cooperative management of invasive species in human-mediated landscapes. American Journal of Agricultural Economics 97(1), 180-198.

Epanchin-Niell, R. S. and J. E. Wilen (2012). Optimal spatial control of biological invasions. Journal of Environmental Economics and Management 63(2), 260270.

Escapa, M. and M. Gutièrrez (1997). Distribution of potential gains from international environmental agreements: The case of the greenhouse effect. Journal of Environmental Economics and Management 33, 1-16. 
Fenichel, E. P., T. J. Richards, , and D. W. Shanafelt (2014). The control of invasive species on private property with neighbor-to-neighbor spillovers. Environmental and Resource Economics, 1-25.

Finnoff, D., A. Potapov, and M. Lewis (2010). Control and the management of a spreading invader. Resource and Energy Economics 32, 534-550.

Geoffard, P.-Y. and T. Philipson (1997). Disease eradication: Private versus public vaccination. The American Economic Review 87(1), 222-230.

Gersovitz, M. and J. Hammer (2004). The economical control of infectious diseases. The Economic Journal 14, 1-27.

Gersovitz, M. and J. Hammer (2005). Tax/subsidy policies toward vector-borne infectious diseases. Journal of Public Economics 89(4), 647-674.

Grimsrud, K., J. Chermak, J. Hansen, J. Thacher, and K. Krause (2008). A two-agent dynamic model with an invasive weed diffusion externality: an application to yellow starthistle (Centaurea solstitialis L.) in new mexico. Journal of Environmental Management 89, 322-335.

Jackson, M. O. and Y. Zenou (2014). Games on networks. Handbook of game theory 4 .

Jorgensen, S., G. Martín-Herrán, and G. Zaccour (2010). Dynamic games in the economics and management of pollution. Environmental Modeling and Assessment 15(6), 433-467.

Kaffine, D. T. and C. Costello (2011). Unitization of spatially connected renewable resources. The BE Journal of Economic Analysis $\&$ Policy 11(1).

Kaiser, B. and K. Burnett (2010). Spatial economic analysis of early detection and rapid response strategies for an invasive species. Resource and Energy Economics 32, 566-585.

Kaitala, V. and M. Pohjola (1988). Optimal recovery of a shared resource stock: A differential game model with efficient memory equilibria. Natural Resource Modeling 3, 91-119.

Leung, B., D. M. Lodge, D. Finnoff, J. F. Shogren, M. A. Lewis, and G. Lamberti (2002). An ounce of prevention or a pound of cure: bioeconomic risk analysis of invasive species. Proceedings of the Royal Society of London. Series B: Biological Sciences 269(1508), 2407-2413. 
Lichtenberg, E. and D. Zilberman (1986). The econometrics of damage control: why specification matters. American Journal of Agricultural Economics 68, 261-273.

Mäler, K.-G. (1989). The acid rain. In H. Folmer and E. van Terland (Eds.), Valuation Methods and Policy Making in Environmental Economics, pp. 23152. Elsevier Science Publishers.

Mäler, K. G. and A. de Zeeuw (1998). The acid rain differential game. Environmental and Resource Economics 12(2), 167-184.

Nathan, R., G. G. Katul, H. S. Horn, S. M. Thomas, R. Oren, R. Avissar, S. W. Pacala, and S. A. Levin (2002). Mechanisms of long-distance dispersal of seeds by wind. Nature 418(6896), 409-413.

Olson, L. and D. Roy (2002). The economics of controlling a stochastic biological invasion. American Journal of Agricultural Economics 84, 1311-1316.

Palfrey, R. and J. Prisbrey (1996). Altruism, reputation and noise in linear public goods experiments. Journal of Public Economics 61, 409-427.

Pimentel, D. (2011). Biological Invasions. CRC press.

Regan, T., M. McCarthy, P. Baxter, F. Panetta, and H. Possingham (2006). Optimal eradication: when to stop looking for an invasive plant. Ecological Letters 9, $759-766$.

Reinganum, J. and N. Stokey (1985). Oligopoly extraction of a common property natural resource: The importance of the period of commitment in dynamic games. International Economic Review 26(1), 161-173.

Rowthorn, R. E., R. Laxminarayan, and C. Gilligan (2009). Optimal control of epidemics in metapopulations. Journal of the Royal Society Interface 6(41), $1135-1144$.

Sanchirico, J. N. and J. E. Wilen (1999). Bioeconomics of spatial exploitation in a patchy environment. Journal of Environmental Economics and Management 37(2), 129-150.

Shapiro, C. (1982). Consumer information, product quality, and seller reputation. The Bell Journal of Economics 13, 20-35.

Shogren, J. (2000). Risk reductions strategies against the "explosive invader". In W. M. D. S. Perrings, C. (Ed.), The Economics of Biological Invasions, pp. 56-69. Edward Elgar, Northhampton, MA. 
Siegel, D., B. Kinlan, B. Gaylord, and S. Gaines (2003). Lagrangian descriptions of marine larval dispersion. Marine Ecology Progress Series 260, 83-96.

Simberloff, D. (2009). We can eliminate invasions or live with them. successful management projects. Biological Invasions 11, 149-157.

van der Ploeg, F. and A. de Zeeuw (1992). International aspects of pollution control. Environmental and Resource Economics 2(2), 117-139.

Weitzman, M. (2015). A voting architecture for the governance of free-driver externalities, with application to geoengineering. Scandinavian Journal of Economics (Forthcoming).

Wiemer, C. (1987). Optimal disease control through the combined use of preventive and curative measures. Journal of Development Economics 25(2), 301-319.

Winfree, J. and J. McCluskey (2005). Collective reputation and quality. American Journal of Agricultural Economics 87, 206-213. 


\section{Appendix}

\section{Proof of Proposition 1}

Denoting $\mathbf{x}_{t}$ and $\mathbf{e}_{t}$ the period $t$ vectors of state and residual stock levels, and using the Bellman equation (4) the optimality conditions require

$$
-k_{i}\left(e_{i t}\right)+c_{i}\left(e_{i t}\right)-\delta\left(\sum_{j=1}^{N} \frac{\partial V_{i t+1}\left(x_{t+1}\right)}{\partial x_{j t+1}} \cdot \frac{\partial x_{j t+1}}{\partial e_{i t}}\right)=0 \text { for } i=1, \ldots, N
$$

The first two terms in (27) are independent of $\mathbf{x}_{t}$ by inspection. The derivative of the value function in period $t+1$ depends on the period $t+1$ state, but is independent of the period $t$ state. For an interior solution we have $e_{i t}<x_{i t}$ and, using (1), we conclude that $x_{i t+1}$ depends on $e_{i t}$ but not on $x_{i t}$. Therefore, the terms in the bracket in condition (27) are independent of $\mathbf{x}_{t}$. Thus the period $t$ game problem has state independent strategies: $\hat{e}_{i t}$ does not depend on $\mathbf{x}_{t}$ for any $i=1, \ldots, N$. Using 11 yields $\frac{\partial x_{j t+1}}{\partial e_{i t}}=D_{i j} g^{\prime}\left(e_{i t}\right)$ for any $j=1, \ldots, N$ and we obtain

$$
\frac{\partial V_{i t+1}\left(x_{t+1}\right)}{\partial x_{i t+1}}=c_{i}\left(x_{i t+1}\right), \quad \frac{\partial V_{i t+1}\left(x_{t+1}\right)}{\partial x_{j t+1}}=0 \text { for } j \neq i
$$

Substituting this expression and that of $\frac{\partial x_{j t+1}}{\partial e_{i t}}$ into 27 we obtain 5 . This necessary condition is also sufficient provided the assumption of convexity of returns in the strategy (residual stock level) is satisfied: the second order condition $\left(\mathrm{SOC}_{i}\right)$ is

$$
S O C_{i}=k_{i}^{\prime}\left(\hat{e}_{i t}\right)-c_{i}^{\prime}\left(\hat{e}_{i t}\right)+\delta D_{i i}\left[g^{\prime \prime}\left(\hat{e}_{i t}\right) c_{i}\left(\hat{x}_{i t+1}\right)+D_{i i}\left(g^{\prime}\left(\hat{e}_{i t}\right)\right)^{2} c_{i}^{\prime}\left(\hat{x}_{i t+1}\right)\right]>0 .
$$

Finally, since the biological growth function is independent of time, as is spread, self retention and economic costs, the optimal choice, $\hat{e}_{i t}$, is independent of time for any $i=1, \ldots, N$.

\section{Proof of Proposition 2}

Denote $\Psi_{i} \equiv k_{i}\left(e_{i t}\right)-c_{i}\left(e_{i t}\right)+\delta c_{i}\left(x_{i t+1}\right) D_{i i} g^{\prime}\left(e_{i t}\right)=0$ the first order condition (FOC) defining owner $i$ 's best response and $e_{i t}\left(e_{j t}, \ldots, e_{l t}\right)$ owner $i$ 's reaction function: we know that $\Psi_{i}\left[e_{i t}\left(e_{j t}, \ldots, e_{l t}\right), e_{j t}, \ldots, e_{l t}\right] \equiv 0$. Differentiating (omitting the time argument), we get:

$$
\begin{aligned}
& \frac{\partial \Psi_{i}}{\partial e_{i}} \frac{\partial e_{i}}{\partial e_{j}}+\frac{\partial \Psi_{i}}{\partial e_{j}}=0 \Leftrightarrow \frac{\partial e_{i}}{\partial e_{j}}=-\frac{\frac{\partial \Psi_{i}}{\partial e_{j}}}{\frac{\partial \Psi_{i}}{\partial e_{i}}} \\
\text { with } \quad & \frac{\partial \Psi_{i}}{\partial e_{i}}=k_{i}^{\prime}\left(\hat{e}_{i t}\right)-c_{i}^{\prime}\left(\hat{e}_{i t}\right)+\delta D_{i i}\left[c_{i}\left(\hat{x}_{i t+1}\right) g^{\prime \prime}\left(\hat{e}_{i t}\right)+c_{i}^{\prime}\left(\hat{x}_{i t+1}\right) D_{i i}\left(g^{\prime}\left(\hat{e}_{i t}\right)\right)^{2}\right]=S O C_{i}>0 \\
& \frac{\partial \Psi_{i}}{\partial e_{j}}=\delta D_{i i} D_{j i} c^{\prime}\left(x_{i t+1}\right) g^{\prime}\left(e_{i t}\right) g^{\prime}\left(e_{j t}\right)<0 \quad \text { since } c^{\prime}(.)<0 \text { and } g^{\prime}(.)>0
\end{aligned}
$$

\section{Proof of Proposition 3}

Due to the Bellman equation (4) the first order conditions for a corner solution require:

$$
k_{i}(0)-c_{i}(0)+\delta\left(\sum_{j=1}^{N} \frac{\partial V_{i t+1}(\mathbf{0})}{\partial x_{j t+1}} \cdot \frac{\partial x_{j t+1}}{\partial e_{i t}}\right) \geq 0 \text { for } i=1, \ldots, N .
$$


The first two terms in 29 are independent of $\mathbf{x}_{t}$ by inspection. The derivative of the value function in period $t+1$ depends on the period $t+1$ state, but is independent of the period $t$ state. For a corner solution we have $\hat{e}_{i t}=0<x_{i t}$ and, using (1), we conclude that $x_{i t+1}=0$ does not depend on $x_{i t}$. Therefore, the terms in the bracket in (29) are independent of $\mathbf{x}_{t}$. This implies that the period $t$ game problem has state independent strategies: $\hat{e}_{i t}=0$ does not depend on $\mathbf{x}_{t}$ for any $i=1, \ldots, N$. Using $[1]$ yields $\frac{\partial x_{j t+1}}{\partial e_{i t}}=D_{i j} g^{\prime}(0)$ for any $j=1, \ldots, N$ and we obtain

$$
\frac{\partial V_{i t+1}\left(x_{t+1}\right)}{\partial x_{i t+1}}=c_{i}(0), \quad \frac{\partial V_{i t+1}\left(x_{t+1}\right)}{\partial x_{j t+1}}=0 \text { for } j \neq i
$$

Substituting this expression and that of $\frac{\partial x_{j t+1}}{\partial e_{i t}}$ into $\sqrt{29}$ we obtain condition $\sqrt{6}$. This necessary condition is also sufficient provided the assumption of convexity of costs in the strategy (residual stock level) is satisfied. The second order condition $\left(S O C_{i}\right)$ is:

$$
k_{i}^{\prime}(0)-c_{i}^{\prime}(0)+\delta D_{i i}\left[g^{\prime \prime}(0) c_{i}(0)+D_{i i}\left(g^{\prime}(0)\right)^{2} c_{i}^{\prime}(0)\right]>0
$$

Finally, the optimal choice $\hat{e}_{i t}=0$ is obviously time independent for any $i=1, \ldots, N$. Now, if global eradication and interior equilibria co-exist, global eradication is Pareto-dominant and coordination will ensure its emergence. For any property $i$, denoting $\Pi_{i}^{g e}$ and $\Pi_{i}^{i n t}$ the payoffs resulting from global eradication and interior equilibria, we have:

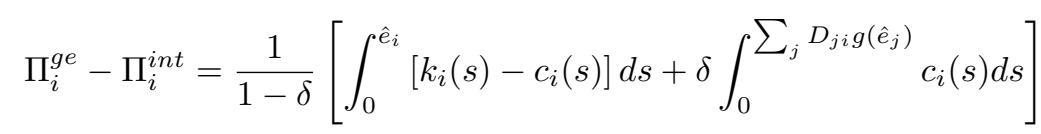

and thus (since $\left.\sum_{j} D_{j i} g\left(\hat{e}_{j}\right) \geq D_{i i} g\left(\hat{e}_{i}\right)\right)$ :

$$
\begin{aligned}
\Pi_{i}^{g e}-\Pi_{i}^{i n t} & >\frac{\left[\int_{0}^{\hat{e}_{i}}\left[k_{i}(s)-c_{i}(s)\right] d s+\delta \int_{0}^{D_{i i} g\left(\hat{e}_{i}\right)} c_{i}(s) d s\right]}{1-\delta} \\
& =\frac{\int_{0}^{\hat{e}_{i}}\left[k_{i}(s)-c_{i}(s)+\delta D_{i i} g^{\prime}(s) c_{i}\left(D_{i i} g(s)\right)\right] d s}{1-\delta}
\end{aligned}
$$

Finally, since $f_{i}(s)=k_{i}(s)-c_{i}(s)+\delta D_{i i} g^{\prime}(s) c_{i}\left(D_{i i} g(s)\right)$ is non-decreasing on $\left[0, \hat{e}_{i}\right]$ by the assumption of convexity of costs in the strategy:

$\Pi_{i}^{g e}-\Pi_{i}^{i n t}>\frac{\int_{0}^{\hat{e}_{i}}\left[k_{i}(s)-c_{i}(s)+\delta D_{i i} g^{\prime}(s) c_{i}\left(D_{i i} g(s)\right)\right] d s}{1-\delta} \geq \frac{\hat{e}_{i}}{1-\delta}\left[k_{i}(0)-c_{i}(0)+\delta D_{i i} g^{\prime}(0) c_{i}(0)\right]>0$

\section{Proof of the claim in Remark 1}

The first condition ensures that global eradication is optimal. The second condition implies that, for any $0<e_{j}<x_{j 0}(j \neq i)$ we have, for any $\left.e \in\right] 0, x_{i 0}[$ :

$$
\begin{aligned}
& k_{i}(e)-c_{i}(e)+\delta D_{i i} g^{\prime}(e) c_{i}\left(D_{i i} g(e)+\sum_{j \neq i} D_{j i} g\left(e_{j}\right)\right)>k_{i}(e)-c_{i}(e)+\delta D_{i i} g^{\prime}(e) c_{i}\left(D_{i i} g(e)+\sum_{j \neq i} D_{j i} g\left(x_{j 0}\right)\right) \\
& \geq k_{i}(0)-c_{i}(0)+\delta D_{i i} g^{\prime}(0) c_{i}\left(\sum_{j \neq i} D_{j i} g\left(x_{j 0}\right)\right) \geq 0
\end{aligned}
$$

Thus condition (5) cannot be satisfied by any $e<x_{i 0}$, which concludes the proof. 


\section{Proof of Proposition 4}

The strategies described in Proposition 4 form a MPNE by combining the proofs of Propositions 1 (for the interior choices) and 3 (for the corner solutions). Time and state independencies follow similarly. We provide the description of the main arguments. Since 6 is not satisfied, the convexity of the payoff functions implies that at least one property owner has incentives to increase the residual stock level compared to full eradication (when other owners eradicate the entire stock in their properties). Thus global eradication is not an equilibrium outcome. If the owner of property $j \notin E$ decides at period $t$ to increase the residual stock level (again when it is zero in any property $i \in E$, and positive in any property $l \notin E$ ) her optimal choice is $\hat{e}_{j t}$ (as characterized in (8)). Then (7) implies that the owner of property $i \in E$ will keep the residual stock level at zero (when the owner of property $j \notin E$ chooses $\hat{e}_{j t}$ ). Thus, at the equilibrium, the residual stock level will be zero on property $i \in E$ and positive on property $j \notin E$.

\section{Proof of Proposition 5}

This follows from dynamic programming arguments similar to those in Proposition 1 (using first order conditions related to the social welfare function ensuring an interior policy in any property $i$ ).

\section{Proof of Proposition 6}

Condition 11 follows from dynamic programming arguments similar to those in Proposition 3 (using first order condition related to the social welfare function ensuring $\bar{e}_{i}=0$ in any property i). The proof of case (b) follows from arguments similar to those of Proposition 4

\section{Proof of Proposition 7}

If $D_{i i}=1$, then (5) and (10) are identical. If $D_{i i}=0$, then (5) becomes $c_{i}\left(\hat{e}_{i t}\right)-k_{i}\left(\hat{e}_{i t}\right)=0$, while 100 becomes $c_{i}\left(\bar{e}_{i t}\right)-k_{i}\left(\bar{e}_{i t}\right)=\delta \sum_{j \neq i} D_{i j} c_{j}\left(\bar{x}_{j t+1}\right) g^{\prime}\left(\bar{e}_{i t}\right)>0$. The LHS of (5) and 100 are similar. Since $c_{i}^{\prime}\left(e_{i t}\right)-k_{i}^{\prime}\left(e_{i t}\right)<0$, for these equalities to hold, we must have $\bar{e}_{i t}<\hat{e}_{i t}$.

We now examine the case where $D_{i i} \in(0,1)$ by comparing Equations 5 and 10 . Rewriting gives:

$$
\begin{aligned}
& k_{i}\left(\hat{e}_{i t}\right)=c_{i}\left(\hat{e}_{i t}\right)-\delta c_{i}\left(\hat{x}_{i t+1}\right) D_{i i} g^{\prime}\left(\hat{e}_{i t}\right) \\
& k_{i}\left(\bar{e}_{i t}\right)=c_{i}\left(\bar{e}_{i t}\right)-\delta c_{i}\left(\bar{x}_{i t+1}\right) D_{i i} g^{\prime}\left(\bar{e}_{i t}\right)-\underbrace{\delta \sum_{j \neq i} c_{j}\left(\bar{x}_{j t+1}\right) D_{i j} g^{\prime}\left(\bar{e}_{i t}\right)}_{\mathcal{L}} .
\end{aligned}
$$

The first two terms on the right hand side of (32) and (33) are identical. Since $k_{i}($.$) is increasing$ in $e$, and because $\mathcal{L} \geq 0$, it is clear that $\hat{e}_{i t} \geq \bar{e}_{i t}$, with equality only if $D_{i j}=0 \forall j \neq i$.

\section{Proof of Proposition 8}

In a case with two properties $A$ and $B$, assuming interior equilibria, we have:

$$
\begin{aligned}
& k_{A}\left(e_{A}\right)=c_{A}\left(e_{A}\right)-\delta c_{A}\left(x_{A}\right) D_{A A} g^{\prime}\left(e_{A}\right) \\
& k_{B}\left(e_{B}\right)=c_{B}\left(e_{B}\right)-\delta c_{B}\left(x_{B}\right) D_{B B} g^{\prime}\left(e_{B}\right)
\end{aligned}
$$

We omit subscript $t$ in (34) and (35). They imply that $e_{A}$ and $e_{B}$ are the solution to the above system (since $x_{k}=\sum_{l} D_{l k} g\left(e_{l}\right)$ for $k=A, B$ ) and are both functions of $\theta=$ $\left\{D_{A A}, D_{B B}, D_{A B}, D_{B A}\right\}$. These first order conditions are functions of $\theta$ as $\Psi_{A}\left(e_{A}(\theta), e_{B}(\theta), \theta\right) \equiv$ 0 and $\Psi_{B}\left(e_{A}(\theta), e_{B}(\theta), \theta\right) \equiv 0$. We can thus totally differentiate both conditions: 


$$
\left\{\begin{array}{l}
\frac{\partial \Psi_{A}}{\partial e_{A}} \frac{\partial e_{A}}{\partial \theta}+\frac{\partial \Psi_{A}}{\partial e_{B}} \frac{\partial e_{B}}{\partial \theta}+\frac{\partial \Psi_{A}}{\partial \theta}=0 \\
\frac{\partial \Psi_{B}}{\partial e_{A}} \frac{\partial e_{A}}{\partial \theta}+\frac{\partial \Psi_{B}}{\partial e_{B}} \frac{\partial e_{B}}{\partial \theta}+\frac{\partial \Psi_{B}}{\partial \theta}=0
\end{array}\right.
$$

Solving this system, we get that $\frac{\partial e_{A}}{\partial \theta}$ (and symmetrically $\frac{\partial e_{B}}{\partial \theta}$ ):

$$
\frac{\partial e_{A}}{\partial \theta}=\frac{-\frac{\partial \Psi_{A}}{\partial \theta} \frac{\partial \Psi_{B}}{\partial e_{B}}+\frac{\partial \Psi_{B}}{\partial \theta} \frac{\partial \Psi_{A}}{\partial e_{B}}}{\frac{\partial \Psi_{A}}{\partial e_{A}} \frac{\partial \Psi_{B}}{\partial e_{B}}-\frac{\partial \Psi_{A}}{\partial e_{B}} \frac{\partial \Psi_{B}}{\partial e_{A}}}
$$

with $\quad \begin{aligned} \frac{\partial \Psi_{A}}{\partial e_{A}}=S O C_{A}>0, & \frac{\partial \Psi_{A}}{\partial e_{B}}=\delta D_{A A} D_{B A} g^{\prime}\left(e_{A}\right) g^{\prime}\left(e_{B}\right) c_{A}^{\prime}\left(x_{A}\right)<0 \\ \frac{\partial \Psi_{B}}{\partial e_{B}}=S O C_{B}>0, & \frac{\partial \Psi_{B}}{\partial e_{A}}=\delta D_{B B} D_{A B} g^{\prime}\left(e_{A}\right) g^{\prime}\left(e_{B}\right) c_{B}^{\prime}\left(x_{B}\right)<0\end{aligned}$

Table 1: Partial derivatives

\begin{tabular}{|c|c|c|}
\hline$\theta$ & $\frac{\partial \Psi_{A}}{\partial \theta}$ & $\frac{\partial \Psi_{B}}{\partial \theta}$ \\
\hline$D_{A A}$ & $\delta g^{\prime}\left(e_{A}\right)\left[c_{A}\left(x_{A}\right)+D_{A A} c_{A}^{\prime}\left(x_{A}\right) g\left(e_{A}\right)\right]$ & 0 \\
$D_{B A}$ & $\delta D_{A A} g^{\prime}\left(e_{A}\right) c_{A}^{\prime}\left(x_{A}\right) g\left(e_{B}\right)$ & 0 \\
\hline$D_{B B}$ & 0 & $\delta g^{\prime}\left(e_{B}\right)\left[c_{B}\left(x_{B}\right)+D_{B B} c_{B}^{\prime}\left(x_{B}\right) g\left(e_{B}\right)\right]$ \\
\hline$D_{A B}$ & 0 & $\delta D_{B B} g^{\prime}\left(e_{B}\right) c_{B}^{\prime}\left(x_{B}\right) g\left(e_{A}\right)$ \\
\hline
\end{tabular}

The denominator of (37) is $S O C_{A} S O C_{B}-\delta^{2} D_{A A} D_{B B} D_{A B} D_{B A}\left(g^{\prime}\left(e_{A}\right)\right)^{2}\left(g^{\prime}\left(e_{B}\right)\right)^{2} c_{A}^{\prime}\left(x_{A}\right) c_{B}^{\prime}\left(x_{B}\right)$. If either $D_{A A}$ or $D_{B B}$ is sufficiently large, it is positive. This is valid if either $D_{A B}$ or $D_{B A}$ is sufficiently large and residual stocks remain positive, if the discount factor is small enough, or if either cost function is linear $\left(c_{A}^{\prime}()=\right.$.0 or $\left.c_{B}^{\prime}()=0.\right)$. Using Table 1 , we deduce:

$$
\begin{aligned}
& \frac{\partial e_{A}}{\partial D_{A A}}=\frac{\delta g^{\prime}\left(e_{A}\right)\left[c_{A}\left(x_{A}\right)+D_{A A} c_{A}^{\prime}\left(x_{A}\right) g\left(e_{A}\right)\right] S O C_{B}}{S O C_{A} S O C_{B}-\delta^{2} D_{A A} D_{B B} D_{A B} D_{B A}\left(g^{\prime}\left(\hat{e}_{A}\right)\right)^{2}\left(g^{\prime}\left(\hat{e}_{B}\right)\right)^{2} c_{A}^{\prime}\left(\hat{x_{A}}\right) c_{B}^{\prime}\left(\hat{x_{B}}\right)} \\
& \frac{\partial e_{A}}{\partial D_{B A}}=-\frac{\delta D_{A A} g^{\prime}\left(e_{A}\right) g\left(e_{B}\right) c_{A}^{\prime}\left(x_{A}\right) S O C_{B}}{S O C_{A} S O C_{B}-\delta^{2} D_{A A} D_{B B} D_{A B} D_{B A}\left(g^{\prime}\left(e_{A}\right)\right)^{2}\left(g^{\prime}\left(e_{B}\right)\right)^{2} c_{A}^{\prime}\left(x_{A}\right) c_{B}^{\prime}\left(x_{B}\right)} \\
& \frac{\partial e_{A}}{\partial D_{B B}}=\frac{\delta^{2} D_{A A} D_{B A} g^{\prime}\left(e_{A}\right)\left[g^{\prime}\left(e_{B}\right)\right]^{2} c_{A}^{\prime}\left(x_{A}\right)\left[c_{B}\left(x_{B}\right)+D_{B B} c_{B}^{\prime}\left(x_{B}\right) g\left(e_{B}\right)\right]}{S O C_{A} S O C_{B}-\delta^{2} D_{A A} D_{B B} D_{A B} D_{B A}\left(g^{\prime}\left(\hat{e}_{A}\right)\right)^{2}\left(g^{\prime}\left(\hat{e}_{B}\right)\right)^{2} c_{A}^{\prime}\left(\hat{x_{A}}\right) c_{B}^{\prime}\left(\hat{x_{B}}\right)} \\
& \frac{\partial e_{A}}{\partial D_{A B}}=\frac{\delta^{2} D_{A A} D_{B B} D_{B A} g^{\prime}\left(e_{A}\right)\left[g^{\prime}\left(e_{B}\right)\right]^{2} c_{A}^{\prime}\left(x_{A}\right) c_{B}^{\prime}\left(x_{B}\right) g\left(e_{A}\right)}{S O C_{A} S O C_{B}-\delta^{2} D_{A A} D_{B B} D_{A B} D_{B A}\left(g^{\prime}\left(\hat{e}_{A}\right)\right)^{2}\left(g^{\prime}\left(\hat{e}_{B}\right)\right)^{2} c_{A}^{\prime}\left(\hat{x_{A}}\right) c_{B}^{\prime}\left(\hat{x_{B}}\right)}
\end{aligned}
$$

When the denominator is positive $\frac{\partial e_{A}}{\partial D_{B A}}>0$ and $\frac{\partial e_{A}}{\partial D_{A B}}>0$. When the marginal cost is inelastic, i.e. $1>-D_{A A} g\left(e_{A}\right) \frac{c^{\prime}\left(x_{A}\right)}{c\left(x_{A}\right)}>0$, then $\frac{\partial e_{A}}{\partial D_{A A}}<0$. The sign of $\frac{\partial e_{A}}{\partial D_{B B}}$ depends also on the marginal cost elasticity. If it is inelastic, i.e. $1>-D_{B B} g\left(e_{B}\right) \frac{c^{\prime}\left(x_{B}\right)}{c\left(x_{B}\right)}>0$, then $\frac{\partial e_{A}}{\partial D_{B}}<0$. 
Robustness of Proposition 8 when $\sum_{j} D_{i j}=1$

Starting with $D_{A A}$, keeping in mind $D_{A B}=1-D_{A A}$ and $D_{B A}=1-D_{B B}$, we have:

$$
\frac{\partial \hat{e}_{A}}{\partial D_{A A}}=\frac{-\delta g^{\prime}\left(\hat{e}_{A}\right)\left[\left(c_{A}\left(\hat{x}_{A}\right)+D_{A A} g\left(\hat{e}_{A}\right) c_{A}^{\prime}\left(\hat{x}_{A}\right)\right) S O C_{B}+\delta D_{A A} D_{B B} D_{B A}\left(g^{\prime}\left(\hat{e}_{B}\right)\right)^{2} g\left(\hat{e}_{A}\right) c_{A}^{\prime}\left(\hat{x}_{A}\right) c_{B}^{\prime}\left(\hat{x}_{B}\right)\right]}{S O C_{A} S O C_{B}-\delta^{2} D_{A A} D_{B B} D_{A B} D_{B A}\left(g^{\prime}\left(\hat{e}_{A}\right)\right)^{2}\left(g^{\prime}\left(\hat{e}_{B}\right)\right)^{2} c_{A}^{\prime}\left(\hat{x}_{A}\right) c_{B}^{\prime}\left(\hat{x}_{B}\right)} .
$$

The denominator of the right hand side term is positive for large values of either $D_{A A}$ or $D_{B B}$. When $\varepsilon_{A}<1$ the term between brackets in the numerator is positive, and $\frac{\partial \hat{e}_{A}}{\partial D_{A A}}$ is negative. When $\varepsilon_{A}>1$ and $D_{B B}$ is large enough, the term between brackets in the numerator is negative, and $\frac{\partial \hat{e}_{A}}{\partial D_{A A}}$ is positive. Results might differ only when $\varepsilon_{A}>1$ and the value of $D_{A A}$ is sufficiently large, while $D_{B B}$ is moderate:

$$
\left(c_{A}\left(\hat{x}_{A}\right)+D_{A A} g\left(\hat{e}_{A}\right) c_{A}^{\prime}\left(\hat{x}_{A}\right)\right) S O C_{B}+\delta D_{A A} D_{B B} D_{B A}\left(g^{\prime}\left(\hat{e}_{B}\right)\right)^{2} g\left(\hat{e}_{A}\right) c_{A}^{\prime}\left(\hat{x}_{A}\right) c_{B}^{\prime}\left(\hat{x}_{B}\right)>0 .
$$

About the effect of $D_{B A}$, as $D_{A B}=1-D_{A A}$ and $D_{B A}=1-D_{B B}$ we have:

$$
\frac{\partial \hat{e}_{A}}{\partial D_{B A}}=-\frac{\delta D_{A A} g^{\prime}\left(\hat{e}_{A}\right) c_{A}^{\prime}\left(\hat{x}_{A}\right)\left[g\left(\hat{e}_{B}\right) S O C_{B}+\delta D_{B A}\left(g^{\prime}\left(\hat{e}_{B}\right)\right)^{2}\right]\left[D_{B B} g\left(\hat{e}_{B}\right) c_{B}^{\prime}\left(\hat{x}_{B}\right)+c_{B}\left(\hat{x}_{B}\right)\right]}{S O C_{A} S O C_{B}-\delta^{2} D_{A A} D_{B B} D_{A B} D_{B A}\left(g^{\prime}\left(\hat{e}_{A}\right)\right)^{2}\left(g^{\prime}\left(\hat{e}_{B}\right)\right)^{2} c_{A}^{\prime}\left(\hat{x}_{A}\right) c_{B}^{\prime}\left(\hat{x}_{B}\right)} .
$$

The denominator of the right hand side term is positive for large values of either $D_{A A}$ or $D_{B B}$. When $D_{B B}$ is large, since $D_{B A}=1-D_{B B}$ is low , the term between brackets in the numerator is positive, thus $\frac{\partial \hat{e}_{A}}{\partial D_{B A}}$ is positive. When $D_{A A}$ is large, provided $\varepsilon_{B}<1$ the term between brackets in the numerator is positive, and the same follows for $\frac{\partial \hat{e}_{A}}{\partial D_{B A}}$. Results might differ only when $\varepsilon_{B}>1$ and $D_{A A}$ is sufficiently large. Moreover, it requires that $D_{B B}$ be neither too large nor too small:

$$
g\left(\hat{e}_{B}\right) S O C_{B}+\delta\left(g^{\prime}\left(\hat{e}_{B}\right)\right)^{2}\left(1-D_{B B}\right)\left[D_{B B} g\left(\hat{e}_{B}\right) c_{B}^{\prime}\left(\hat{x}_{B}\right)+c_{B}\left(\hat{x}_{B}\right)\right]<0 .
$$

Moving on to the effects of spread $D_{A B}$ and self-retention $D_{B B}$, we deduce that

$$
\frac{\partial \hat{e}_{A}}{\partial D_{A B}}=-\frac{\partial \hat{e}_{A}}{\partial D_{A A}} ; \quad \frac{\partial \hat{e}_{A}}{\partial D_{B B}}=-\frac{\partial \hat{e}_{A}}{\partial D_{B A}} .
$$

Concerning $\frac{\partial \hat{e}_{A}}{\partial D_{A B}}$ Proposition 8 is robust when $D_{B B}$ is sufficiently large, and are reversed in cases when (1) $D_{A A}$ has sufficiently large values, (2) $\varepsilon_{A}>1$ and (3) $\frac{\partial \hat{e}_{A}}{\partial D_{A A}}>0$ is satisfied. The effect of $D_{B B}$ differs already when $D_{B B}$ is large: an increase in $D_{B B}$ always results in a larger residual stock level in property $A$. When $D_{A A}$ is large, the effect is reversed when the following conditions are satisfied: (1) $D_{B B}$ is neither too large nor too small, (2) $\varepsilon_{B}>1$ and (3) $\frac{\partial \hat{e}_{A}}{\partial D_{B A}}<0$ is satisfied.

\section{Proof of Lemma 1}

If global eradication is a Nash equilibrium outcome, then for any $i \in I$, due to Proposition 3 and Condition (11) in Proposition 6 we have $k_{i}(0)-\left[1-\delta D_{i i} g^{\prime}(0)\right] c_{i}(0) \geq 0$. Since $g^{\prime}(0)$ is positive and all spread parameters are non negative, the following condition holds:

$$
k_{i}(0)-\left[1-\delta D_{i i} g^{\prime}(0)\right] c_{i}(0)+\delta g^{\prime}(0) \sum_{j \neq i} D_{i j} c_{j}(0) \geq k_{i}(0)-\left(1-\delta D_{i i} g^{\prime}(0)\right) c_{i}(0) \geq 0 .
$$

Using Propositions 3 and 6 implies that global eradication is socially optimal. 


\section{Proof of Corollary 1}

The result follows from Condition 6 in Proposition 3 and Condition 11 in Proposition 6 .

\section{Proof of Corollary 2}

The result follows from Condition 6 in Proposition 3 and Condition 11 in Proposition 6 .

\section{Proof of Proposition 9}

From Corollary 2 the interval characterizing values of marginal abatement costs (on property i) over which tensions arise is $\left[\frac{k_{i}(0)}{1-\delta D_{i i} g^{\prime}(0)}, \frac{k_{i}(0)+\delta g^{\prime}(0) \sum_{j \neq i} D_{i j} c_{j}(0)}{1-\delta D_{i i} g^{\prime}(0)}\right]$, and its size is given by

$$
\Delta_{i}=\frac{k_{i}(0)+\delta g^{\prime}(0) \sum_{j \neq i} D_{i j} c_{j}(0)}{1-\delta D_{i i} g^{\prime}(0)}-\frac{k_{i}(0)}{1-\delta D_{i i} g^{\prime}(0)}=\frac{\delta g^{\prime}(0) \sum_{j \neq i} D_{i j} c_{j}(0)}{1-\delta D_{i i} g^{\prime}(0)} .
$$

First, we have $\frac{\partial \Delta_{i}}{\partial D_{i j}}=\frac{\delta g^{\prime}(0) c_{j}(0)}{1-\delta D_{i i} g^{\prime}(0)}>0$ since $1-\delta D_{i i} g^{\prime}(0)>0$ and provided $c_{j}(0)>0$, which implies that the length of the interval increases as spread increases. Second, differentiating with respect to the self retention rate, we obtain $\frac{\partial \Delta_{i}}{\partial D_{i i}}=\frac{\left(\delta g^{\prime}(0)\right)^{2} \sum_{j \neq i} D_{i j} c_{j}(0)}{1-\delta D_{i i} g^{\prime}(0)}>0$ since $1-\delta D_{i i} g^{\prime}(0)>$ 0 and provided $\sum_{j \neq i} D_{i j} c_{j}(0)>0$. Thus, the length of the interval increases as self retention increases (provided that self retention remains lower than $\frac{1}{\delta g^{\prime}(0)}$ ).

\section{Proof of Proposition 10}

For large marginal dynamic costs, global eradication is an equilibrium. From Proposition 3 it solves the coordination problem (if there is any). As global eradication is also socially optimal (by lemma 1), the gains from cooperation equal zero. For moderate dynamic marginal costs, the global eradication equilibrium does not exist, while global eradication is socially optimal. Provided that an equilibrium is interior, the gains from cooperation are then:

$$
\Delta^{c}=\sum_{i=1}^{N}\left(\bar{\Pi}_{i}-\hat{\Pi}_{i}\right)=\frac{1}{1-\delta} \sum_{i=1}^{N}\left[\int_{0}^{\hat{e}_{i}}\left[k_{i}(s)-c_{i}(s)\right] d s+\delta \int_{0}^{\sum_{j} D_{j i} g\left(\hat{e}_{j}\right)} c_{i}(s) d s\right]
$$

Function $\Psi\left(e_{1}, \ldots, e_{N}\right)=\sum_{i=1}^{N}\left[\int_{0}^{e_{i}}\left[k_{i}(s)-c_{i}(s)\right] d s+\delta \int_{0}^{\sum_{j} D_{j i} g\left(e_{j}\right)} c_{i}(s) d s\right]$ is convex due to the convexity of costs in the vector of residual stock levels, thus $\Psi\left(\hat{e}_{1}, \ldots, \hat{e}_{N}\right) \geq \Psi(\mathbf{0})+\sum_{i=1}^{N} \frac{\partial \Psi}{\partial e_{i}}(\mathbf{0}) \hat{e}_{i}$, or $\Delta^{c} \geq \frac{1}{1-\delta}\left(\sum_{i=1}^{N} \hat{e}_{i}\left\{k_{i}(0)-c_{i}(0)+\delta g^{\prime}(0)\left[\sum_{l=1}^{N} D_{j l} c_{l}(0)\right]\right\}\right)$. For low marginal dynamic costs, the socially optimal and decentralized outcomes are interior. The gains from cooperation are $\Delta^{c}=\frac{1}{1-\delta} \sum_{i=1}^{N}\left[\int_{\bar{e}_{i}}^{\hat{e}_{i}}\left[k_{i}(s)-c_{i}(s)\right] d s+\delta \int_{\sum_{j} D_{j i} g\left(\bar{e}_{i}\right)}^{\sum_{D_{i}} D_{j} g\left(\hat{e}_{i}\right)} c_{i}(s) d s\right]$ or $\Delta^{c}=\frac{1}{1-\delta}[\Psi(\hat{\mathbf{e}})-\Psi(\overline{\mathbf{e}})]$, and there is $\left(e_{1}, \ldots, e_{N}\right)$ such that $\left.e_{i} \in\right] \bar{e}_{i}, \hat{e}_{i}\left[\right.$ for any $i \in I$ and $\Psi(\hat{\mathbf{e}})-\Psi(\overline{\mathbf{e}})=\sum_{i=1}^{N}\left(\hat{e}_{i}-\bar{e}_{i}\right) \frac{\partial \Psi}{\partial e_{i}}\left(e_{1}, \ldots, e_{N}\right)$. We deduce (since $\Psi($.$) is increasing in e_{i}$ and decreasing in other arguments) that $\frac{\partial \Psi}{\partial e_{i}}\left(e_{1}, \ldots, e_{N}\right)<$ $\frac{\partial \Psi}{\partial e_{i}}\left(\hat{e}_{i}, \bar{e}_{-i}\right)$ and so:

$$
\frac{\partial \Psi}{\partial e_{i}}\left(e_{1}, \ldots, e_{N}\right)<\delta g^{\prime}\left(\hat{e}_{i}\right) \sum_{j=1}^{N} D_{i j} c_{j}\left(D_{i j} g\left(\hat{e}_{i}\right)+\sum_{l \neq i} D_{l j} g\left(\bar{e}_{l}\right)\right)<\delta g^{\prime}(0) \sum_{j=1}^{N} D_{i j} c_{j}(0) .
$$

Thus $\Delta^{c}<\frac{1}{1-\delta} \sum_{i=1}^{N}\left(\hat{e}_{i}-\bar{e}_{i}\right)\left[\delta g^{\prime}(0) \sum_{j=1}^{N} D_{i j} c_{j}(0)\right]<\frac{1}{1-\delta} \sum_{i=1}^{N}\left(\hat{e}_{i}-\bar{e}_{i}\right)\left[c_{i}(0)-k_{i}(0)\right]$, where the second inequality follows from the assumption. 


\section{Proof of Proposition 11}

We compute the gains from cooperation by the following expression:

$$
\begin{aligned}
S & =\bar{\Pi}_{A}+\bar{\Pi}_{B}-\hat{\Pi}_{A}-\hat{\Pi}_{B} \\
& =\frac{\delta}{1-\delta}\left[\int_{0}^{D_{B A} g\left(\hat{e}_{B}\right)} c_{A}(s) d s+\int_{0}^{\hat{e}_{B}} k_{B}(s) d s+\int_{\hat{e}_{B}}^{D_{B B} g\left(\hat{e}_{B}\right)} c_{B}(s) d s\right]-\int_{0}^{\hat{e}_{B}} c_{B}(s) d s+\int_{0}^{\hat{e}_{B}} k_{B}(s) d s,
\end{aligned}
$$

which is positive by inspection. Moreover, it is easily checked that:

$$
\begin{aligned}
& \bar{\Pi}_{A}-\hat{\Pi}_{A}=\frac{\delta}{1-\delta} \int_{0}^{D_{B A} g\left(\hat{e}_{B}\right)} c_{A}(s) d s>0 \\
& \bar{\Pi}_{B}-\hat{\Pi}_{B}=-\int_{0}^{\hat{e}_{B}} c_{B}(s) d s+\int_{0}^{\hat{e}_{B}} k_{B}(s) d s+\frac{\delta}{1-\delta}\left[\int_{0}^{\hat{e}_{B}} k_{B}(s) d s+\int_{\hat{e}_{B}}^{D_{B B} g\left(\hat{e}_{B}\right)} c_{B}(s) d A q\right)
\end{aligned}
$$

Using Expression 42 and the convexity of costs, we conclude that

$$
\bar{\Pi}_{B}-\hat{\Pi}_{B}<\frac{\hat{e}_{B}}{1-\delta}\left[k_{B}\left(\hat{e}_{B}\right)-c_{B}\left(\hat{e}_{B}\right)+\delta D_{B B} g^{\prime}\left(\hat{e}_{B}\right) c_{B}\left(D_{B B} g\left(\hat{e}_{B}\right)\right)\right] .
$$

The right hand side of (43) equals zero by (19): owner $A(B)$ gains (loses) from cooperation. As gains are positive overall, owner $A$ is willing to induce $B$ to lower her residual stock.

\section{Proof of Proposition 12}

The scheme induces global eradication if the owners' payoffs under side payments are at least those without side-payment. When transfers are allowed, we have:

$$
\begin{aligned}
& \tilde{\Pi}_{A}\left(x_{A t}, e_{A t}, e_{B t}\right)=-\left[\int_{0}^{e_{A t}} k_{A}(s) d s+\int_{e_{A t}}^{x_{A t}} c_{A}(s) d s+\tau\left(e_{A t}, e_{B t}\right)+\Gamma_{t}\right] \\
& \tilde{\Pi}_{B}\left(x_{B t}, e_{A t}, e_{B t}\right)=-\left[\int_{0}^{e_{B t}} k_{B}(s) d s+\int_{e_{B t}}^{x_{B}} c_{B}(s) d s-\tau\left(e_{A t}, e_{B t}\right)-\Gamma_{t}\right]
\end{aligned}
$$

where $\Gamma_{0}=\gamma$ and $\Gamma_{t}=0$ for $t \geq 1$. Using the Bellman equations, the optimality conditions correspond to one outcome: global eradication. One can check that it is the equilibrium when:

$$
k_{A}^{\prime}(e)-c_{A}^{\prime}(e)+\delta D_{A A} g^{\prime \prime}(e) c_{A}\left(D_{A A} g(e)\right)+\delta\left(D_{A A}\right)^{2}\left(g^{\prime}(e)\right)^{2} c_{A}^{\prime}\left(D_{A A} g(e)\right)>0 .
$$

If $B$ deviates, he chooses a decision rule stipulating $e_{B}>0$ when stock level is $x_{B 0}$ (otherwise the game ends at $t=0$ ). This cannot be profitable. We first consider a deviation stipulating $e_{B}>0$ when stock level is $x_{B 0}$, and to eradicate otherwise: $B$ deviates at $t=0$ only. We have:

$$
\begin{aligned}
\tilde{\Pi}_{B}(0,0)-\tilde{\Pi}_{B}^{d} & =\int_{0}^{e_{B}} k_{B}(s) d s-\int_{0}^{e_{B}} c_{B}(s) d s-\left(k_{B}(0)-c_{B}(0)\right) e_{B}+\delta \int_{0}^{D_{B B} g\left(e_{B}\right)} c_{B}(s) d s \\
& =\int_{0}^{e_{B}}\left[k_{B}(s)-c_{B}(s)+\delta D_{B B} g^{\prime}(s) c_{B}\left(D_{B B} g(s)\right)\right] d s-\left(k_{B}(0)-c_{B}(0)\right) e_{B} .
\end{aligned}
$$

Noticing that $\delta D_{B B} g^{\prime}(e) c_{B}\left(D_{B B} g(e)\right)>0$ for any $e \geq 0$ we deduce

$$
\int_{0}^{e_{B}}\left[k_{B}(s)-c_{B}(s)+\delta D_{B B} g^{\prime}(s) c_{B}\left(D_{B B} g(s)\right)\right] d s>\int_{0}^{e_{B}}\left[k_{B}(s)-c_{B}(s)\right] d s .
$$


As $\phi_{B}(e)=\int_{0}^{e}\left[k_{B}(s)-c_{B}(s)\right] d s$ is strictly convex $\left(\right.$ since $k_{B}^{\prime}(e)>0$ and $\left.c_{B}^{\prime}(e)<0\right)$ :

$$
\int_{0}^{e_{B}}\left[k_{B}(s)-c_{B}(s)\right] d s-\left(k_{B}(0)-c_{B}(0)\right) e_{B}>\left(k_{B}(0)-c_{B}(0)\right) e_{B}-\left(k_{B}(0)-c_{B}(0)\right) e_{B}=0,
$$

which proves our claim. Now it suffices to notice that any strategy stipulating positive residual stocks at $t=0$ (say $e_{B}^{\prime}$ ) and at subsequent periods is dominated by a unique deviation at $t=0$ stipulating residual stock $e_{B}^{\prime}$. If now $A$ deviates, he chooses a decision rule stipulating $e_{A}>0$ when stock level is $x_{A 0}$. This cannot be profitable. We first consider the deviation stipulating $e_{A}>0$ when stock level is $x_{A 0}$, and to eradicate otherwise: $A$ deviates at $t=0$ only. We have:

$$
\begin{aligned}
\tilde{\Pi}_{A}(0,0)-\tilde{\Pi}_{A}^{d} & =\int_{0}^{e_{A}} k_{A}(s) d s-\int_{0}^{e_{A}} c_{A}(s) d s+\delta \int_{0}^{D_{A A} g\left(e_{A}\right)} c_{A}(s) d s+\left[k_{A}(0)-c_{A}(0)+\delta D_{A A} g^{\prime}(0) c_{A}(0)\right] e_{A} \\
& =\int_{0}^{e_{A}}\left[k_{A}(s)-c_{A}(s)+\delta D_{A A} g^{\prime}(s) c_{A}\left(D_{A A} g(s)\right)\right] d s+\left[k_{A}(0)-c_{A}(0)+\delta D_{A A} g^{\prime}(0) c_{A}(0)\right] e_{A} .
\end{aligned}
$$

By $444 \phi_{A}(e)=\int_{0}^{e}\left[k_{A}(s)-c_{A}(s)+\delta D_{A A} g^{\prime}(s) c_{A}\left(D_{A A} g(s)\right)\right] d s$ is convex, and

$\tilde{\Pi}_{A}(0,0)-\tilde{\Pi}_{A}^{d} \geq\left[k_{A}(0)-c_{A}(0)+\delta D_{A A} g^{\prime}(0) c_{A}(0)\right] e_{A}+\left[k_{A}(0)-c_{A}(0)+\delta D_{A A} g^{\prime}(0) c_{A}(0)\right] e_{A}>0$,

which follows since 18 holds. This proves our claim. As for $B$, any strategy stipulating positive residual stocks at $t=0$ and at subsequent periods is dominated by a deviation at $t=0$ only. We now show that both owners can be (weakly) better off compared to the case of no transfer. Due to $(18)$ and $\sqrt{19}$ the outcome of the game without side-payment is partial eradication and payoffs $\Pi_{A}$ and $\hat{\Pi}_{B}$ are defined by 20 and 21 . Define $K_{A}=\tilde{\Pi}_{A}(0,0)-\hat{\Pi}_{A}=\bar{\Pi}_{A}-\gamma-\hat{\Pi}_{A}$ and $K_{B}=\tilde{\Pi}_{B}(0,0)-\hat{\Pi}_{B}=\bar{\Pi}_{B}+\gamma-\hat{\Pi}_{B}$ the difference between owners' payoffs when they exchange payments, and when no transfer is allowed. Proposition 11 shows that $\bar{\Pi}_{A}-\hat{\Pi}_{A}>0$ while $\bar{\Pi}_{B}-\hat{\Pi}_{B}<0$. Global eradication is induced when $K_{i} \geq 0$ for $i=A, B$, that is:

$$
\bar{\Pi}_{A}-\hat{\Pi}_{A} \geq \gamma \text { and } \gamma \geq \hat{\Pi}_{B}-\bar{\Pi}_{B} \Longrightarrow \hat{\Pi}_{B}-\bar{\Pi}_{B} \leq \gamma \leq \bar{\Pi}_{A}-\hat{\Pi}_{A} .
$$

Since $\hat{\Pi}_{B}-\bar{\Pi}_{B}-\left(\bar{\Pi}_{A}-\hat{\Pi}_{A}\right)=\hat{\Pi}_{A}+\hat{\Pi}_{B}-\left(\bar{\Pi}_{A}+\bar{\Pi}_{B}\right)<0$ by Proposition 11 any value of $\gamma \in\left[\hat{\Pi}_{B}-\bar{\Pi}_{B}, \bar{\Pi}_{A}-\hat{\Pi}_{A}\right]$ makes both owners (weakly) better off with side payments.

\section{Proof of Proposition 13}

We obtain (keeping in mind that $\frac{\partial \hat{e}_{B}}{\partial D_{B A}}=0$ ):

$$
\begin{aligned}
\frac{\partial S}{\partial D_{B A}} & =\frac{\delta}{1-\delta} g\left(\hat{e}_{B}\right) c_{A}\left(D_{B A} g\left(\hat{e}_{B}\right)\right)>0 . \\
\frac{\partial S}{\partial D_{B B}} & =\frac{\delta}{1-\delta}\left[D_{B A} g^{\prime}\left(\hat{e}_{B}\right) c_{A}\left(D_{B A} g\left(\hat{e}_{B}\right)\right) \frac{\partial \hat{e}_{B}}{\partial D_{B B}}+g\left(\hat{e}_{B}\right) c_{B}\left(D_{B B} g\left(\hat{e}_{B}\right)\right)\right],
\end{aligned}
$$

with

$$
\frac{\partial \hat{e}_{B}}{\partial D_{B B}}=\frac{-\delta g^{\prime}\left(\hat{e}_{B}\right)\left[g^{\prime}\left(\hat{e}_{B}\right) D_{B B} c_{B}^{\prime}\left(D_{B B} g\left(\hat{e}_{B}\right)\right)+c_{B}\left(D_{B B} g\left(\hat{e}_{B}\right)\right)\right]}{S O C_{B}} .
$$

As in Proposition $8 \frac{\partial \hat{e}_{B}}{\partial D_{B B}}>0$ if and only of the marginal cost is elastic. Third, we differentiate 26 with respect to $D_{B A}$, and we obtain (keeping in mind that $\frac{\partial \hat{B}_{B}}{\partial D_{B A}}=0$ ):

$$
\frac{\partial \gamma}{\partial D_{B A}}=\frac{2 \delta}{1-\delta} g\left(\hat{e}_{B}\right) c_{A}\left(D_{B A} g\left(\hat{e}_{B}\right)\right)>0 .
$$


Finally, we differentiate 26 with respect to $D_{B B}$ :

$$
\frac{\partial \gamma}{\partial D_{B B}}=\frac{\delta}{2(1-\delta)}\left[\frac{\delta}{1-\delta}\left[D_{B A} g^{\prime}\left(\hat{e}_{B}\right) c_{A}\left(D_{B A} g\left(\hat{e}_{B}\right)\right) \frac{\partial \hat{e}_{B}}{\partial D_{B B}}-g\left(\hat{e}_{B}\right) c_{B}\left(D_{B B} g\left(\hat{e}_{B}\right)\right)\right],\right]
$$

By Proposition $8 \frac{\partial \hat{e}_{B}}{\partial D_{B B}}<0$ if and only of the marginal cost is inelastic, so $\frac{\partial \gamma}{\partial D_{B B}}<0$. Otherwise $\frac{\partial \hat{e}_{B}}{\partial D_{B B}}<0$, and the sign of $\frac{\partial \gamma}{\partial D_{B B}}$ is ambiguous and depends on $\frac{\partial\left(\bar{\Pi}_{A}-\hat{\Pi}_{A}\right)}{\partial D_{B B}} \gtreqless \frac{\partial\left(\hat{\Pi}_{B}-\bar{\Pi}_{B}\right)}{\partial D_{B B}}$.

The impact of a "small" initial invasion

We focus on the case of $N=2$ to keep the exposition simple. A first result will be useful:

Proposition 14. Consider two properties, $A$ and $B$, in which $x_{A 0}>\bar{e}_{A}$ and $x_{B 0}=0<\bar{e}_{B}$, where $\bar{e}_{A}$ and $\bar{e}_{B}$ are characterized by (5). Moreover, assume that condition (11) does not hold (for both properties), and denote $\left.e_{A 0} \in\right] 0, x_{A 0}[$ the solution to:

$$
k_{A}(e)-c_{A}(e)+\delta g^{\prime}(e)\left[D_{A A} c_{A}\left(D_{A A} g(e)\right)+D_{A B} c_{B}\left(D_{A B} g(e)\right)\right]=0 .
$$

Then, if $D_{A A} g\left(e_{A 0}\right)>\bar{e}_{A}$ and $D_{A B} g\left(e_{A 0}\right)>\bar{e}_{B}$ hold, the socially optimal outcome is:

$$
\bar{e}_{A 0}=e_{A 0}, \quad \bar{e}_{B 0}=0 ; \quad \forall t \geq 1 \quad \bar{e}_{A t}=\bar{e}_{A}, \quad \bar{e}_{B t}=\bar{e}_{B} .
$$

We now state formally the claim made in Section 7

Proposition 15. Assume that there are two properties, $A$ and B, and consider: (1) Case 1, which is consistent with Proposition 14; (2) Case 2, in which $x_{A 0}>\bar{e}_{A}$ and $x_{B 0}>\bar{e}_{B}$, where $\bar{e}_{A}$ and $\bar{e}_{B}$ are characterized by (5). Then the socially optimal outcome in both cases is identical starting at $t=1$, while at $t=0$ :

$$
\bar{e}_{A 0}^{2}=\bar{e}_{A}>e_{A 0}=\bar{e}_{A 0}^{1},
$$

where $\bar{e}_{A 0}^{i}$ denotes the socially optimal residual stock level at the initial period in case $i=1,2$.

Compared to case 1 , the invasion is more spatially-extensive in case 2: initial stock in $A$ is identical in both cases, and is lower in $B$ in case 1 . Yet, the overall control strategy at $t=0$ is more aggressive in case 1 : the residual stocks in $t=0$ are lower in both properties. A similar result obtains under non-cooperative management. The proofs highlight that assumptions $D_{A A} g\left(e_{A 0}\right)>\bar{e}_{A}$ and $D_{A B} g\left(e_{A 0}\right)>\bar{e}_{B}$ in Proposition 14 are not crucial. The exposition is simple since the socially optimal outcomes are identical starting from $t=1$. Weakening this assumption would result in more aggressive socially optimal controls in case 1 for at least $t=0$ and $t=1$.

\section{Supplementary material}

\section{Proof of the claim in Footnote 9}

The first condition (and the convexity of present value costs) and the second condition imply respectively that, for any $\left.\left.e_{j} \in\right] 0, x_{j 0}\right](j \neq i)$, we have:

$$
\begin{array}{r}
k_{i}(0)-c_{i}(0)+\delta D_{i i} g^{\prime}(0) c_{i}\left(\sum_{j \neq i} D_{j i} g\left(e_{j}\right)\right)<k_{i}(0)-c_{i}(0)+\delta D_{i i} g^{\prime}(0) c_{i}(0)<0, \\
k_{i}\left(x_{i 0}\right)-c_{i}\left(x_{i 0}\right)+\delta D_{i i} g^{\prime}\left(x_{i 0}\right) c_{i}\left(D_{i i} g\left(x_{i 0}\right)+\right. \\
\left.\sum_{j \neq i} D_{j i} g\left(e_{j}\right)\right)>k_{i}\left(x_{i 0}\right)-c_{i}\left(x_{i 0}\right) \\
+\delta D_{i i} g^{\prime}\left(x_{i 0}\right) c_{i}\left(\sum_{j \in I} D_{j i} g\left(x_{j 0}\right)\right) \geq 0
\end{array}
$$


The first inequality follows from $c_{i}^{\prime}()<$.0 while the second follows from assumptions. Conditions (48) and (49) imply that there is $\left.e_{i} \in\right] 0, x_{i 0}$ [ such that

$$
k_{i}\left(e_{i}\right)-c_{i}\left(e_{i}\right)+\delta D_{i i} g^{\prime}\left(e_{i}\right) c_{i}\left(D_{i i} g\left(e_{i}\right)+\sum_{j \neq i} D_{j i} g\left(e_{j}\right)\right)=0 .
$$

With continuously differentiable functions, the Brouwer fixed point theorem implies that there is $0<\hat{e}_{i}<x_{i 0}$ such that $\left(\hat{e}_{1}, \ldots, \hat{e}_{N}\right)$ is characterized by the optimality conditions. Provided $g^{\prime}\left(\hat{e}_{i}\right) \geq \frac{1}{\delta D_{i i}}$ for any $i \in I$, this vector is such that $\hat{x}_{i}=\sum_{j} D_{j i} g\left(\hat{e}_{j}\right)>\hat{e}_{i}$ and this concludes the proof by using Proposition 1. If this is not the case, there is $j \in I$ such that $\hat{x}_{j} \leq \hat{e}_{j}$ : thus $c_{j}\left(\hat{x}_{j}\right) \geq c_{j}\left(\hat{e}_{j}\right)$ and then, using the optimality condition characterizing $\hat{e}_{j}$, we have:

$$
c_{j}\left(\hat{e}_{j}\right) \leq c_{j}\left(\hat{x}_{j}\right) \leq \delta D_{j j} g^{\prime}\left(\hat{e}_{j}\right) c_{j}\left(\hat{x}_{j}\right)=c_{j}\left(\hat{e}_{j}\right)-k_{j}\left(\hat{e}_{j}\right)<c_{j}\left(\hat{e}_{j}\right)
$$

which is a contradiction. This concludes the proof. We provide sufficient existence conditions: if $g^{\prime}(e) \geq 1$ (which holds for logistic functions) then the condition on $g^{\prime}\left(\hat{e}_{i}\right)$ is not required.

\section{Proof of the claim in Footnote 19}

Assume that the assumptions of Corollary 1 are satisfied. If there is $i \in I$ such that either $c_{i}(0) \leq k_{i}(0)$ or $\sum_{j \neq i} D_{j i} g\left(x_{j 0}\right) \leq c_{i}^{-1}\left(c_{i}(0)-k_{i}(0)\right)$ holds, then an interior equilibrium does not exist. Indeed, a sufficient condition for non existence is that there is a property (say $i$ ) for which:

$$
k_{i}(0)-c_{i}(0)+\delta D_{i i} g^{\prime}(0) c_{i}\left(\sum_{j \neq i} D_{j i} g\left(x_{j 0}\right)\right) \geq 0 .
$$

Thus we obtain, for any $\left(e_{1}, \ldots, e_{N}\right)$ such that $0<e_{i}<x_{i 0}$ for any $i$ :

$$
\begin{aligned}
& k_{i}\left(e_{i}\right)-c_{i}\left(e_{i}\right)+\delta D_{i i} g^{\prime}\left(e_{i}\right) c_{i}\left(D_{i i} g\left(e_{i}\right)+\sum_{j \neq i} D_{j i} g\left(e_{j}\right)\right)>k_{i}\left(e_{i}\right)-c_{i}\left(e_{i}\right)+\delta D_{i i} g^{\prime}\left(e_{i}\right) c_{i}\left(D_{i i} g\left(e_{i}\right)\right) \\
& \left.+\sum_{j \neq i} D_{j i} g\left(x_{j 0}\right)\right) \geq k_{i}(0)-c_{i}(0)+\delta D_{i i} g^{\prime}(0) c_{i}\left(\sum_{j \neq i} D_{j i} g\left(x_{j 0}\right)\right) \geq 0 .
\end{aligned}
$$

The first inequality follows from $c_{i}^{\prime}()<$.0 and the second one from the convexity of costs. Coming back to condition 50 and rewriting, we obtain $\delta D_{i i} g^{\prime}(0) \geq \frac{c_{i}(0)-k_{i}(0)}{c_{i}\left(\sum_{j \neq i} D_{j i} g\left(x_{j 0}\right)\right)}$. If $c_{i}(0)-k_{i}(0) \leq 0$ then this inequality is satisfied, and this concludes the proof of the first case. Second, using Corollary 1 a sufficient condition is then $1 \geq \frac{c_{i}(0)-k_{i}(0)}{c_{i}\left(\sum_{j \neq i} D_{j i} g\left(x_{j 0}\right)\right)}$ or $\sum_{j \neq i} D_{j i} g\left(x_{j 0}\right) \leq c_{i}^{-1}\left(c_{i}(0)-k_{i}(0)\right)$ and this concludes the proof, since a sufficient condition here is that there is a property $i$ such that $x_{k 0} \leq g^{-1}\left(\frac{1}{\sum_{j \neq i} D_{j i}}\left(c_{i}\right)^{-1}\left(c_{i}(0)-k_{i}(0)\right)\right)$ is satisfied, with $x_{k 0}=\max _{j \neq i} x_{j 0}$.

\section{Proof of Proposition 14 (Stated in Appendix)}

Using the dynamic programming equation and deriving the optimality conditions for an interior policy starting at period $t=1$, we obtain:

$$
\begin{aligned}
& k_{A}\left(\bar{e}_{A 1}\right)-c_{A}\left(\bar{e}_{A 1}\right)+\delta g^{\prime}\left(\bar{e}_{A 1}\right)\left[D_{A A} c_{A}\left(D_{A A} g\left(\bar{e}_{A 1}\right)+D_{B A} g\left(\bar{e}_{B 1}\right)\right)+D_{A B} c_{B}\left(D_{B B} g\left(\bar{e}_{B 1}\right)+D_{A B} g\left(\bar{e}_{A 1}\right)\right)\right]=0 \\
& k_{B}\left(\bar{e}_{B 1}\right)-c_{B}\left(\bar{e}_{B 1}\right)+\delta g^{\prime}\left(\bar{e}_{B 1}\right)\left[D_{B B} c_{B}\left(D_{B B} g\left(\bar{e}_{B 1}\right)+D_{A B} g\left(\bar{e}_{A 1}\right)\right)+D_{B A} c_{A}\left(D_{A A} g\left(\bar{e}_{A 1}\right)+D_{B A} g\left(\bar{e}_{B 1}\right)\right)\right]=0
\end{aligned}
$$

Convexity of costs in the vector of residual stock levels ensures that they are necessary and sufficient: an interior solution at $t=1$ results in $\bar{e}_{A 1}=\bar{e}_{A}$ and $\bar{e}_{B 1}=\bar{e}_{B}$. Since at $t=1$ we 
have $D_{A A} g\left(e_{A 0}\right)>\bar{e}_{A}$ and $D_{A B} g\left(e_{A 0}\right)>\bar{e}_{B}$, such residual stocks are feasible and constitute the interior solution. Provided conditions of Proposition 1 are satisfied $\bar{x}_{i}=D_{i i} g\left(\bar{e}_{i}\right)+D_{j i} g\left(\bar{e}_{j}\right)>\bar{e}_{i}$ for $i, j=A, B$ and $i \neq j$. The choice of $\bar{e}_{A}$ and $\bar{e}_{B}$ is thus interior at any $t \geq 1$ and satisfies the optimality condition: $\bar{e}_{i t}=\bar{e}_{i}$ for any $i=A, B$ and any $t \geq 1$. Now at $t=0$ : For $B$, the optimal residual stock is obviously $\bar{e}_{B 0}=0=x_{B 0}$. Regarding $A$, accounting for the interior policy path starting at $t=1$, if the optimal residual stock at $t=0$ is interior $\left(x_{A 0}>\bar{e}_{A 0}>0\right)$ we have:

$$
k_{A}\left(\bar{e}_{A 0}\right)-c_{A}\left(\bar{e}_{A 0}\right)+\delta g^{\prime}\left(\bar{e}_{A 0}\right)\left[D_{A A} c_{A}\left(D_{A A} g\left(\bar{e}_{A 0}\right)\right)+D_{A B} c_{B}\left(D_{A B} g\left(\bar{e}_{A 0}\right)\right)\right]=0,
$$

which is also sufficient by the convexity of costs: thus $\bar{e}_{A 0}=e_{A 0}$. We now show that $0<e_{A 0}<$ $x_{A 0}$. First, $e_{A 0}>0$ since $k_{A}(0)-c_{A}(0)+\delta g^{\prime}(0)\left[D_{A A} c_{A}(0)+D_{A B} c_{B}(0)\right]<0$. Second, since $x_{A 0}>\bar{e}_{A}$ the convexity of costs implies:

$$
\begin{aligned}
& k_{A}\left(x_{A 0}\right)-c_{A}\left(x_{A 0}\right)+\delta g^{\prime}\left(x_{A 0}\right)\left[D_{A A} c_{A}\left(D_{A A} g\left(x_{A 0}\right)\right)+D_{A B} c_{B}\left(D_{A B} g\left(x_{A 0}\right)\right)\right] \\
& >k_{A}\left(x_{A 0}\right)-c_{A}\left(x_{A 0}\right)+\delta g^{\prime}\left(x_{A 0}\right)\left[D_{A A} c_{A}\left(D_{A A} g\left(x_{A 0}\right)+D_{B A} g\left(\bar{e}_{B}\right)\right)+D_{A B} c_{B}\left(D_{A B} g\left(x_{A 0}\right)+D_{B B} g\left(\bar{e}_{B}\right)\right)\right] \\
& \geq k_{A}\left(\bar{e}_{A}\right)-c_{A}\left(\bar{e}_{A}\right)+\delta g^{\prime}\left(\bar{e}_{A}\right)\left[D_{A A} c_{A}\left(D_{A A} g\left(\bar{e}_{A}\right)+D_{B A} g\left(\bar{e}_{B}\right)\right)+D_{A B} c_{B}\left(D_{A B} g\left(\bar{e}_{A}\right)+D_{B B} g\left(\bar{e}_{B}\right)\right)\right]=0,
\end{aligned}
$$

where the first inequality follows from $c_{i}^{\prime}()<$.0 , the second from the convexity of costs, and the final one from the characterization of $\bar{e}_{A}$. Combined with the convexity of costs and continuity of damage and cost functions, it implies that $e_{A 0}$ exists, is unique, and satisfies $0<e_{A 0}<x_{A 0}$.

\section{Proof of Proposition 15 (Stated in Appendix)}

The socially optimal policy in both cases follow from Propositions 6 and 14 . We show that $\bar{e}_{A}>e_{A 0}$ holds: for a given residual stock $e_{A}$ :

$$
\begin{aligned}
& k_{A}\left(e_{A}\right)-c_{A}\left(e_{A}\right)+\delta g^{\prime}\left(e_{A}\right)\left[D_{A A} c_{A}\left(D_{A A} g\left(e_{A}\right)+D_{B A} g\left(\bar{e}_{B}\right)\right)+D_{A B} c_{B}\left(D_{A B} g\left(e_{A}\right)+D_{B B} g\left(\bar{e}_{B}\right)\right)\right] \\
& <k_{A}\left(e_{A}\right)-c_{A}\left(e_{A}\right)+\delta g^{\prime}\left(e_{A}\right)\left[D_{A A} c_{A}\left(D_{A A} g\left(e_{A}\right)\right)+D_{A B} c_{B}\left(D_{A B} g\left(e_{A}\right)\right)\right] .
\end{aligned}
$$

This inequality follows from $c_{A}^{\prime}()<$.0 and this implies that, now taking $e_{A}=\bar{e}_{A}$ :

$$
f\left(\bar{e}_{A}\right):=k_{A}\left(\bar{e}_{A}\right)-c_{A}\left(\bar{e}_{A}\right)+\delta g^{\prime}\left(\bar{e}_{A}\right)\left[D_{A A} c_{A}\left(D_{A A} g\left(\bar{e}_{A}\right)\right)+D_{A B} c_{B}\left(D_{A B} g\left(\bar{e}_{A}\right)\right)\right]>0 .
$$

Since we have by definition of $e_{A 0}$ that $f\left(e_{A 0}\right)=0$, we deduce the inequality $f\left(\bar{e}_{A}\right)>f\left(e_{A 0}\right)$, which implies $\bar{e}_{A}>e_{A 0}$ as $f($.$) is increasing by the assumption of convexity of costs.$

\section{Is global eradication Pareto-dominant?}

The proof of Proposition 3 shows that global eradication Pareto dominates an interior equilibrium; the arguments apply to partial eradication outcomes too. Pareto-dominance of global eradication holds generically, a sufficient condition is: for any $i \in I$ we have

$$
k_{i}(0) \geq c_{i}(0) \max \left(\frac{1}{1+\delta}, \frac{1+\delta-\delta D_{i i} g^{\prime}(0)}{1+\delta}\right)
$$

This condition is stronger than condition $k_{i}(0)-c_{i}(0)+\delta D_{i i} g^{\prime}(0) c_{i}(0)>0$ for any $i \in I$ (more or less depending on the discount factor and the self retention parameter). The proof is as follows. If (51) holds for any $i \in I$ then, considering another MPNE, it necessarily results in a positive residual stock at $t=0$, otherwise the game ends and global eradication obtains. If $e_{i 0}$ denotes the residual stock in property $i$ corresponding to this MPNE:

$\Pi_{i}^{g e}-\Pi_{i}=\int_{0}^{e_{i 0}}\left[k_{i}(s)-c_{i}(s)\right] d s+\delta\left[\int_{0}^{e_{i 1}}\left[k_{i}(s)-c_{i}(s)\right] d s+\int_{0}^{\sum_{j} D_{j i} g\left(e_{i 0}\right)} c_{i}(s) d s+\delta V_{i 2}\left(\mathbf{x}_{2}\right)\right]$ 
where $e_{i 1} \geq 0$ denotes the period-1 residual stock level related to this MPNE, and $V_{i 2}\left(\mathbf{x}_{2}\right) \geq 0$ its present-value cost starting at $t=2$ (which is non-negative). This implies that

$$
\begin{aligned}
\Pi_{i}^{g e}-\Pi_{i} & \geq \int_{0}^{e_{i 0}}\left[k_{i}(s)-c_{i}(s)\right] d s+\delta \int_{0}^{\sum_{j} D_{j i} g\left(e_{j 0}\right)} c_{i}(s) d s+\delta \int_{0}^{e_{i 1}}\left[k_{i}(s)-c_{i}(s)\right] d s \\
& =\int_{0}^{e_{i 0}}\left[k_{i}(s)-c_{i}(s)\right] d s+\delta\left[\int_{0}^{\sum_{j} D_{j i} g\left(e_{j 0}\right)} c_{i}(s) d s-\int_{0}^{e_{i 1}} c_{i}(s) d s\right]+\delta \int_{0}^{e_{i 1}} k_{i}(s) d s
\end{aligned}
$$

And since $e_{i 1} \leq x_{i 1}=\sum_{j} D_{j i} g\left(e_{j 0}\right)$ by definition and $c_{i}(e)>0$ for any $e \in\left[0, \sum_{j} D_{j i} g\left(e_{j 0}\right)\right]$ (assuming $c_{i}(e)$ is well behaved at $e=0$, that is $c_{i}(0)<\infty$ ) we deduce:

$$
\Pi_{i}^{g e}-\Pi_{i} \geq \int_{0}^{e_{i 0}}\left[k_{i}(s)-c_{i}(s)\right] d s+\delta \int_{0}^{e_{i 1}} k_{i}(s) d s .
$$

Now since functions $f_{i}(s)=k_{i}(s)-c_{i}(s)$ and $k_{i}(s)$ are increasing, this inequality implies:

$$
\Pi_{i}^{g e}-\Pi_{i}>e_{i 0}\left[k_{i}(0)-c_{i}(0)\right]+\delta e_{i 1} k_{i}(0)
$$

When $e_{i 1} \geq e_{i 0}$ we deduce $\Pi_{i}^{g e}-\Pi_{i}>e_{i 0}\left[(1+\delta) k_{i}(0)-c_{i}(0)\right] \geq 0$ by condition (51). Now, if $0 \leq e_{i 1}<e_{i 0}$ is satisfied, coming back to $52 p$ we have, since $\sum_{j} D_{j i} g\left(e_{j 0}\right) \geq D_{i i} g\left(e_{i 0}\right)$ :

$$
\Pi_{i}^{g e}-\Pi_{i} \geq \int_{0}^{e_{i 0}}\left[k_{i}(s)-c_{i}(s)\right] d s+\delta \int_{0}^{D_{i i} g\left(e_{i 0}\right)} c_{i}(s) d s+\delta \int_{0}^{e_{i 1}}\left[k_{i}(s)-c_{i}(s)\right] d s
$$

Finally, since $f_{i}(s)=k_{i}(s)-c_{i}(s)$ and $m_{i}(s)=k_{i}(s)-c_{i}(s)+\delta D_{i i} g^{\prime}(s) c_{i}\left(D_{i i} g(s)\right)$ are increasing $\left(m_{i}(\right.$.$) is increasing due to the convexity of costs in the strategy), we have:$

$$
\Pi_{i}^{g e}-\Pi_{i}>e_{i 0}\left[k_{i}(0)-c_{i}(0)+\delta D_{i i} g^{\prime}(0) c_{i}(0)\right]+\delta e_{i 1}\left[k_{i}(0)-c_{i}(0)\right]
$$

When $k_{i}(0)<c_{i}(0)$ (otherwise the conclusion is straightforward) we deduce, since $e_{i 1}<e_{i 0}$ :

$\Pi_{i}^{g e}-\Pi_{i}>e_{i 0}\left(k_{i}(0)-c_{i}(0)+\delta D_{i i} g^{\prime}(0) c_{i}(0)+\delta\left[k_{i}(0)-c_{i}(0)\right]\right)=e_{i 0}\left[(1+\delta)\left[k_{i}(0)-c_{i}(0)\right]+\delta D_{i i} g^{\prime}(0) c_{i}(0)\right]$

and the right hand side of the inequality is positive by (51), which concludes the proof when $e_{i 1}<e_{i 0}$ is satisfied. To conclude the proof overall, notice that, if a property owner $j$ finds it optimal to choose $e_{j 0}=x_{j 0}$ at $t=0$, then $\Pi_{j}^{g e}>\Pi_{j}$ holds necessarily. Indeed, using the general expression of the owner's payoff, and rewriting condition (52) we obtain:

$\Pi_{j}^{g e}-\Pi_{j} \geq \int_{0}^{x_{j 0}}\left[k_{j}(s)-c_{j}(s)\right] d s+\delta\left[\int_{0}^{\sum_{l \neq j} D_{l j} g\left(e_{l 0}\right)+D_{j j} g\left(x_{j 0}\right)} c_{j}(s) d s-\int_{0}^{e_{j 1}} c_{j}(s) d s\right]+\delta \int_{0}^{e_{j 1}} k_{j}(s) d s$ and the reasoning above yields that $\Pi_{j}^{g e}-\Pi_{j}>0$ holds again. 